\title{
The MUCHFUSS project - searching for hot subdwarf binaries with massive unseen companions
}

\section{Survey, target selection and atmospheric parameters ${ }^{\star, \star \star}$}

\author{
S. Geier ${ }^{1}$, H. Hirsch ${ }^{1}$, A. Tillich ${ }^{1}$, P. F. L. Maxted ${ }^{2}$, S. J. Bentley ${ }^{2}$, R. H. Østensen ${ }^{3}$, U. Heber ${ }^{1}$, B. T. Gänsicke ${ }^{4}$, \\ T. R. Marsh ${ }^{4}$, R. Napiwotzki ${ }^{5}$, B. N. Barlow ${ }^{6}$, and S. J. O’Toole ${ }^{1,7}$ \\ 1 Dr. Karl Remeis-Observatory \& ECAP, Astronomical Institute, Friedrich-Alexander University Erlangen-Nuremberg, \\ Sternwartstr. 7, 96049 Bamberg, Germany \\ e-mail: geier@sternwarte.uni-erlangen.de \\ 2 Astrophysics Group, Keele University, Staffordshire, ST5 5BG, UK \\ 3 Institute of Astronomy, K.U.Leuven, Celestijnenlaan 200D, 3001 Heverlee, Belgium \\ 4 Department of Physics, University of Warwick, Conventry CV4 7AL, UK \\ 5 Centre of Astrophysics Research, University of Hertfordshire, College Lane, Hatfield AL10 9AB, UK \\ 6 Department of Physics and Astronomy, University of North Carolina, Chapel Hill, NC 27599-3255, USA \\ 7 Australian Astronomical Observatory, PO Box 296, Epping, NSW, 1710, Australia
}

Received 1 July 2010 / Accepted 19 March 2011

\begin{abstract}
The project Massive Unseen Companions to Hot Faint Underluminous Stars from SDSS (MUCHFUSS) aims at finding sdBs with compact companions like supermassive white dwarfs $\left(M>1.0 M_{\odot}\right)$, neutron stars or black holes. The existence of such systems is predicted by binary evolution theory and recent discoveries indicate that they are likely to exist in our Galaxy.

A determination of the orbital parameters is sufficient to put a lower limit on the companion mass by calculating the binary mass function. If this lower limit exceeds the Chandrasekhar mass and no sign of a companion is visible in the spectra, the existence of a massive compact companion is proven without the need for any additional assumptions. We identified about 1100 hot subdwarf stars from the SDSS by colour selection and visual inspection of their spectra. Stars with high velocities have been reobserved and individual SDSS spectra have been analysed. In total 127 radial velocity variable subdwarfs have been discovered. Binaries with high RV shifts and binaries with moderate shifts within short timespans have the highest probability of hosting massive compact companions. Atmospheric parameters of 69 hot subdwarfs in these binary systems have been determined by means of a quantitative spectral analysis. The atmospheric parameter distribution of the selected sample does not differ from previously studied samples of hot subdwarfs. The systems are considered the best candidates to search for massive compact companions by follow-up time resolved spectroscopy.
\end{abstract}

Key words. binaries: spectroscopic - subdwarfs

\section{Introduction}

Subluminous B stars (sdBs) are core helium-burning stars with very thin hydrogen envelopes and masses around $0.5 M_{\odot}$ (Heber 1986). A large fraction of the sdB stars are members of short period binaries (Maxted et al. 2001; Napiwotzki et al. 2004a). After the discovery of close binary subdwarfs, several studies aimed at determining the fraction of hot subdwarfs residing in such systems. Samples of hot subdwarfs checked for radial velocity (RV)

* Based on observations at the Paranal Observatory of the European Southern Observatory for programme number 081.D-0819. Based on observations at the La Silla Observatory of the European Southern Observatory for programme number 082.D-0649. Based on observations collected at the Centro Astronómico Hispano Alemán (CAHA) at Calar Alto, operated jointly by the Max-Planck Institut für Astronomie and the Instituto de Astrofísica de Andalucía (CSIC). Based on observations with the William Herschel Telescope operated by the Isaac Newton Group at the Observatorio del Roque de los Muchachos of the Instituto de Astrofisica de Canarias on the island of La Palma, Spain.

$\star \star$ Tables 2-4 and Appendix A are available in electronic form at http://www. aanda.org variations imply the binary fraction ranges from $39 \%$ to $78 \%$ (e.g. Maxted et al. 2001; Napiwotzki et al. 2004a). The orbital periods of subdwarf binaries for which orbital parameters could be determined range from 0.07 to $>10 \mathrm{~d}$ with a peak at $0.5-1.0 \mathrm{~d}$ (e.g. Edelmann et al. 2005; Morales-Rueda et al. 2003a).

For close binary sdBs common envelope ejection is the most probable formation channel (Han et al. 2002, 2003). In this scenario two main sequence stars of different masses evolve in a binary system. The more massive one will reach the red giant phase first and fill its Roche lobe near the tip of the red-giant branch. If the mass transfer to the companion is dynamically unstable, a common envelope is formed. Friction causes the two stellar cores to lose orbital energy, which is deposited within the envelope, and the period of the binary decreases. Eventually, the common envelope is ejected, and a close binary system is formed containing a core helium-burning $\mathrm{sdB}$ and a main sequence companion. A binary consisting of a main sequence star and a white dwarf (WD) may evolve to a close binary sdB with a white dwarf companion in a similar way. Tight constraints can be placed on the nature of the sdB companions only in the rare cases where the systems show eclipses or other features indicative 
of a companion in their light curves (see the catalogue of Ritter \& Kolb 2009, and references therein).

Subdwarf binaries with massive WD companions are candidates for SN Ia progenitors because these systems lose angular momentum due to the emission of gravitational waves and start mass transfer. Transfer of mass or the subsequent merger of the system may cause the WD to approach the Chandrasekhar limit, ignite carbon under degenerate conditions, and explode as a SN Ia (Webbink 1984; Iben \& Tutukov 1984). One of the best-known candidate systems for this double degenerate merger scenario is the sdB+WD binary KPD 1930+2752 (Maxted et al. 2000a; Geier et al. 2007). Mereghetti et al. (2009) showed that in the X-ray binary HD 49798 a massive $\left(>1.2 M_{\odot}\right)$ white dwarf accretes matter from a closely orbiting subdwarf $\mathrm{O}$ companion. The predicted amount of accreted material is sufficient for the WD to reach the Chandrasekhar limit. This makes HD 49798 another candidate SN Ia progenitor, should the companion be a C/O white dwarf (Wang et al. 2009). SN Ia play a key role in the study of cosmic evolution since they are utilised as standard candles for determining the cosmological parameters (e.g. Riess et al. 1998; Leibundgut 2001; Perlmutter et al. 1999). Most recently Perets et al. (2010) showed that helium accretion onto a white dwarf may be responsible for a subclass of faint and calcium-rich SN Ib events.

Due to the tidal influence of the companion in close binary systems, the rotation of the primary ${ }^{1}$ becomes synchronised to its orbital motion. In this case it is possible to constrain the mass of the companion, if mass, projected rotational velocity and surface gravity of the sdB are known. Geier et al. (2008, 2010a,b) analysed high resolution spectra of $41 \mathrm{sdB}$ stars in close binaries, half of all systems with known orbital parameters. In 31 cases, the mass and nature of the unseen companions could be constrained. While most of the derived companion masses were consistent with either late main sequence stars or white dwarfs, the compact companions of some sdBs may be either massive white dwarfs, neutron stars (NS) or stellar mass black holes (BH). However, Geier et al. (2010b) also showed that the assumption of orbital synchronisation in close sdB binaries is not always justified and that their sample suffers from huge selection effects.

Binary evolution theory (Podsiadlowski et al. 2002; Pfahl et al. 2003) predicts the existence of $\mathrm{sdB}+\mathrm{NS} / \mathrm{BH}$ systems formed after two phases of unstable mass transfer and one supernova explosion. The predicted fraction of $\mathrm{sdB}+\mathrm{NS} / \mathrm{BH}$ systems ranges from about $1 \%$ to $2 \%$ of the close sdB binaries (Geier et al. 2010b; Yungelson \& Tutukov 2005; Nelemans 2010).

\section{Project overview}

The work of Geier et al. (2010b) indicates that a population of non-interacting binaries with massive compact companions may be present in our Galaxy. The candidate $\mathrm{sdB}+\mathrm{NS} / \mathrm{BH}$ binaries have low orbital inclinations $\left(15-30^{\circ}\right.$, Geier et al. $\left.2010 \mathrm{~b}\right)$, but high inclination systems must exist as well. A lower limit can be placed on the companion mass by determining the orbital parameters and calculating the binary mass function.

$f_{\mathrm{m}}=\frac{M_{\mathrm{comp}}^{3} \sin ^{3} i}{\left(M_{\mathrm{comp}}+M_{\mathrm{sdB}}\right)^{2}}=\frac{P K^{3}}{2 \pi G}$.

\footnotetext{
1 The more massive component of a binary is usually defined as the primary. However, in most close sdB binaries with unseen companions the masses are unknown and it is not possible to decide a priori which component is the most massive one. For this reason we call the visible $\mathrm{sdB}$ component of the binaries the primary throughout this paper.
}

The RV semi-amplitude $K$ and the period $P$ can be derived from the RV curve; the sdB mass $M_{\mathrm{sdB}}$, the companion mass $M_{\text {comp }}$ and the inclination angle $i$ remain free parameters. We adopt $M_{\mathrm{sdB}}=0.47 M_{\odot}$ and $i<90^{\circ}$ to derive a lower limit for the companion mass. Depending on this minimum mass a qualitative classification of the companions' nature is possible in certain cases. For minimum companion masses lower than $0.45 M_{\odot}$ a main sequence companion can not be excluded because its luminosity would be too low to be detectable in the spectra (Lisker et al. 2005). If the minimum companion mass exceeds $0.45 M_{\odot}$ and no spectral signatures of the companion are visible, it must be a compact object. If it exceeds the Chandrasekhar mass and no sign of a companion is visible in the spectra, the existence of a massive compact companion is proven without the need for any additional assumptions. This is possible if such a binary is seen at high inclination. The project Massive Unseen Companions to Hot Faint Underluminous Stars from SDSS ${ }^{2}$ (MUCHFUSS) aims at finding sdBs with compact companions like supermassive white dwarfs $\left(M>1.0 M_{\odot}\right)$, neutron stars or black holes. First results of our follow-up campaign are published in Geier et al. (2011).

There is an interesting spin-off from this project: the same selection criteria we applied to find binaries with massive compact companions are also well-suited to single out hot subdwarf stars with constant high radial velocities in the Galactic halo, which may be extreme population II or even hypervelocity stars. We have coined the term Hyper-MUCHFUSS to refer to this extended project, the first results of which are presented in Tillich et al. (2011).

\section{Target selection}

The high fraction of sdB stars in close binary systems was initially discovered by the detection of RV shifts using time resolved spectroscopy (Maxted et al. 2001). In the past decade, orbital parameters for about 80 of these systems have been determined. We summarize the orbital parameters of all known sdB binaries and give references in Table A.1 (see also Fig. 1).

To the extent that the companion masses of the known $\mathrm{sdB}$ binaries could be constrained, it turned out that most companions should be either late main sequence stars with masses lower than half a solar mass or compact objects like white dwarfs. Targets for spectroscopic follow-up were selected in different ways depending on the specific aims of each project.

For the MUCHFUSS project the target selection is optimised to find massive compact companions in close orbits around $\mathrm{sdB}$ stars. In order to discover rare objects applying the selection criteria explained in the forthcoming sections, a huge initial dataset is necessary. The enormous SDSS database (Data Release 6, DR6) is therefore the starting point for our survey. Best sky coverage is reached in the Northern hemisphere close to the galactic poles. SDSS data are widely used and therefore also well evaluated in terms of errors and accuracy (York et al. 2000; Abazajian et al. 2009). Moreover, they are supplemented by additional spectroscopic observations of appropriate quality from other sources.

\subsection{Colour selection and visual classification}

Hot subdwarfs are found most easily by applying a colour cut to Sloan photometry. All spectra of point sources with colours

\footnotetext{
2 Sloan Digital Sky Survey.
} 


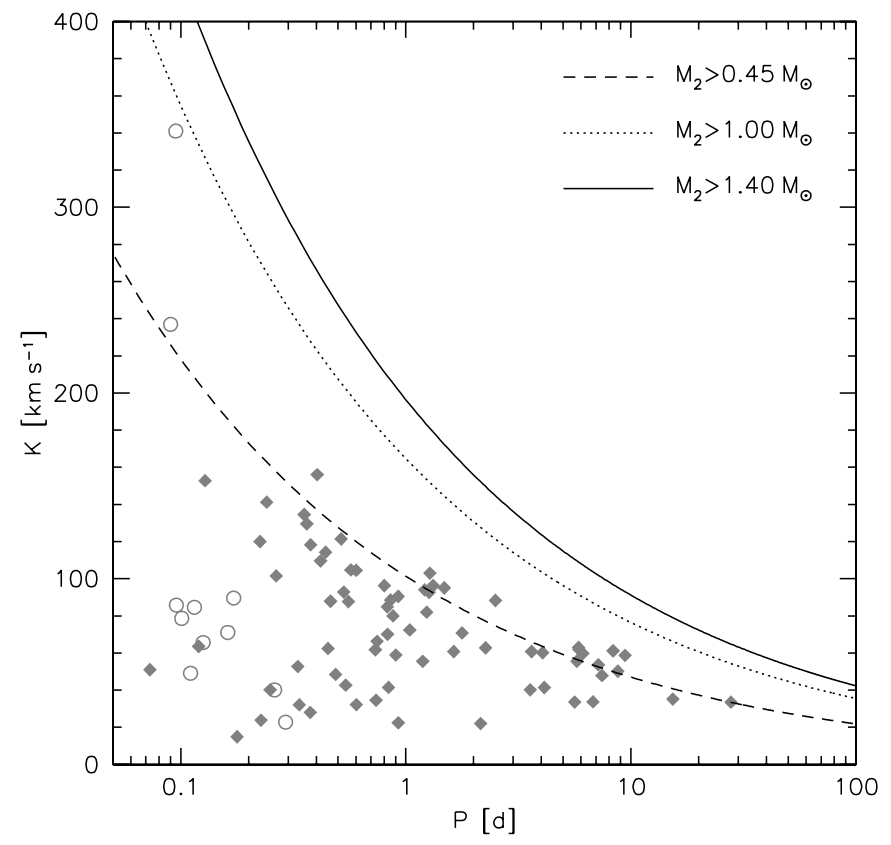

Fig. 1. The RV semiamplitudes of all known sdB binaries with spectroscopic solutions plotted against their orbital periods (see Table A.1). Binaries which were initially discovered in photometric surveys due to indicative features in their light curves (eclipses, reflection effects, ellipsoidal variations) are marked with open circles. Binaries discovered by detection of RV variation from time resolved spectroscopy are marked with filled diamonds. The dashed, dotted and solid lines mark the regions to the right where the minimum companion masses derived from the binary mass function (assuming $0.47 M_{\odot}$ for the sdBs) exceed $0.45 M_{\odot}, 1.00 M_{\odot}$ and $1.40 M_{\odot}$. The two post-RGB objects in the sample have been excluded, because their primary masses are much lower.

$u-g<0.4$ and $g-r<0.1$ were selected. This colour criterion corresponds to a limit in the Johnson photometric system of $U-B<-0.57$ (Jester et al. 2005), similar to the cut-off chosen by UV excess surveys, such as the Palomar Green survey (Green et al. 1986). The corresponding effective temperature of a BHB star is $\simeq 15000 \mathrm{~K}$ (Castelli \& Kurucz 2003), well below the observed range for sdB stars $(>20000 \mathrm{~K})$. The limit of $g-r=+0.1$ corresponds to $B-V=+0.3$ (Jester et al. 2005). This ensures that $\mathrm{sdBs}$ in spectroscopic binaries are included if the dwarf companion is of spectral type $\mathrm{F}$ or later, e.g. the $\mathrm{sdB}+\mathrm{F}$ system $\mathrm{PB} 8783$ at $B-V=+0.13$ and $U-B=-0.65$ (Koen et al. 1997). On the other hand the colour criteria exclude the huge number of QSOs (quasi stellar objects) which were the priority objects of SDSS in the first place. We selected 48267 point sources with spectra in this way.

The spectra from SDSS are flux calibrated and cover the wavelength range from $3800 \AA$ to $9200 \AA$ with a resolution of $R=1800$. Rebassa-Mansergas et al. (2007) verified the wavelength stability to be $<14.5 \mathrm{~km} \mathrm{~s}^{-1}$ from repeat sub-spectra using SDSS observations of F-stars. We obtained the spectra of our targets from the SDSS Data Archive Server ${ }^{3}$ and converted the wavelength scale from vacuum to air. The spectra were classified by visual inspection.

First, we excluded spectra of extragalactic objects and spectra with low quality $(S / N<5)$ or unknown features, leaving us with 10811 spectra of 10153 stars. Figure 2 (left panel) shows

\footnotetext{
${ }^{3}$ das.sdss.org
}

a two-colour plot of all selected objects. To classify the selected spectra, we compared them visually to reference spectra of hot subdwarfs and white dwarfs. Existence, width, and depth of helium and hydrogen absorption lines as well as the flux distribution between 4000 and $6000 \AA$ were used as criteria. Subdwarf B stars show broadened hydrogen Balmer and $\mathrm{He}$ I lines, sdOB stars He II lines in addition, while the spectra of sdO stars are dominated by weak Balmer and strong He II lines depending on the He abundance. A flux excess in the red compared to the reference spectrum as well as the presence of spectral features such as the $\mathrm{Mg}$ I triplet at $5170 \AA$ or the Ca II triplet at $8650 \AA$ were taken as indications of a late type companion (for a few examples see Fig. 3, for spectral classification of hot subdwarf stars see the review by Heber 2009).

Our selection criteria led to a sample containing a total of 1100 hot subdwarfs. 725 belong to the class of single-lined sdBs and sdOBs. Because distinguising between these two subtypes from their spectral appearances alone can be difficult, we decided to treat them as one class. Features indicative of a cool companion were found for 89 of the sdBs. 9 sdOs have main sequence companions, while 198 of them, most of which show helium enrichment, are single-lined. A unique classification was not possible for 79 objects in our sample. Most of these stars are considered candidate sdBs with low temperatures, which cannot be distinguished clearly from blue horizontal branch (BHB) stars or low-mass DA or DB white dwarfs.

Eisenstein et al. (2006) used a semi-automatic method for the spectral classification of white dwarfs and hot subdwarfs from the SDSS DR4, and it is instructive to compare their sample to ours. Our colour cut-off is more restrictive and the confusion limit $(S / N>5)$ is brighter than that of Eisenstein et al. (2006). Due to the redder colour cuts, blue horizontal branch stars enter the Eisenstein et al. sample, which we do not consider as hot subdwarf stars (see Heber 2009). Applying our colour cuts to the hot subdwarf sample of Eisenstein et al. (2006) yields 691 objects. The stars missing in our sample are mostly fainter than $g=19$ mag as expected. Most recently, Kleinman (2010) extended the classifications to the SDSS DR7 and found 1409 hot subdwarf stars. Since no details are published, the sample can not be compared to ours yet. Considering our more restrictive colour cuts and confusion limit, the numbers compare very well with ours. This gives us confidence that our selection method is efficient.

In Fig. 2 (right panel) only the subdwarf stars brighter than $g=18 \mathrm{mag}$ are plotted. With less pollution by poor spectra, two sequences become clearly visible. The solid symbols mark single-lined sdBs and sdOs, while the open squares mark binaries with late type companions of most likely $\mathrm{K}$ and $\mathrm{G}$ type visible in the spectra. The contribution of the cool companions shifts the colours of the stars to the red. As can be seen in Fig. 2 the upper sequence also contains apparently single stars. Since the spectra are not corrected for interstellar reddening, some of these objects may show an excess in the red not due to the presence of a cool companion. Spectral features indicative of a late-type companion and small excesses in the red may have been missed for the faintest targets with the noisiest data.

In Fig. 2 (right panel) we also compare the sample to synthetic colours suitable for hot subdwarf stars. We chose the grid of Castelli \& Kurucz (2003) ${ }^{4}$ and selected models with high gravity $(\log g=5.0)$. The models reproduce the lower envelope of the targets in the colour-colour-diagram very well for effective

\footnotetext{
4 http://wwwuser.oat.ts.astro.it/castelli/colors/ sloan.html
} 

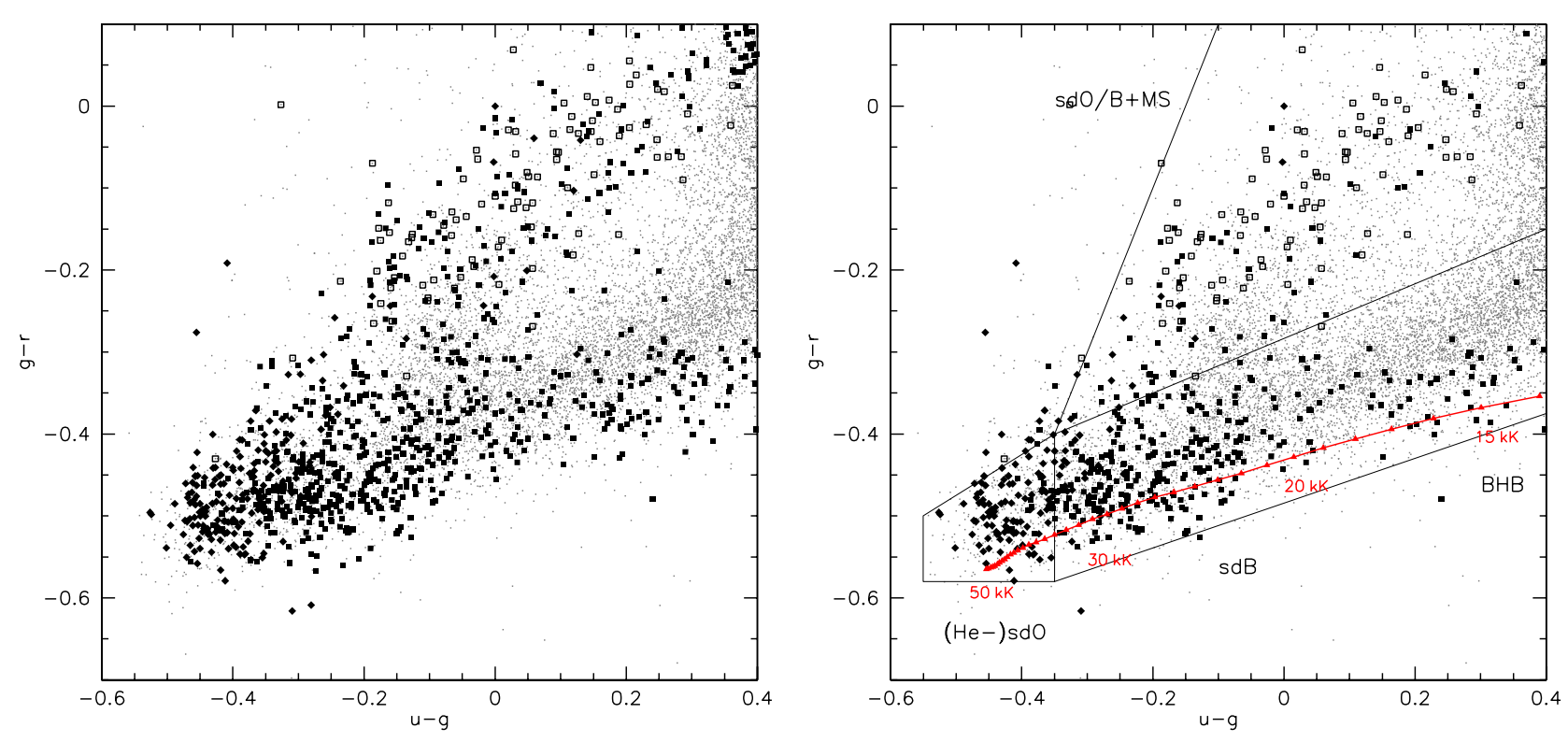

Fig. 2. Left panel. SDSS $g-r$-colours plotted against $u-g$ of all stars. The grey dots mark all stellar objects with spectra available in the SDSS database. Most of them are classified as DA white dwarfs. The solid diamonds mark (He-)sdO stars, the solid squares sdB and sdOB stars. Open squares mark hot subdwarfs with main sequence companions visible in the spectra. Most of these objects are white dwarfs of DA type. Right panel. Only subdwarfs with $g<18$ mag are plotted. The sequence of composite objects is clearly separated from the single-lined stars. Synthetic colours from Castelli \& Kurucz (2003) for stars with temperatures ranging from $14000 \mathrm{~K}$ to $50000 \mathrm{~K}(\log g=5.0)$ are marked with upward triangles and connected. The stepsize of the colour grid is $1000 \mathrm{~K}$. The labels mark models of certain temperatures.

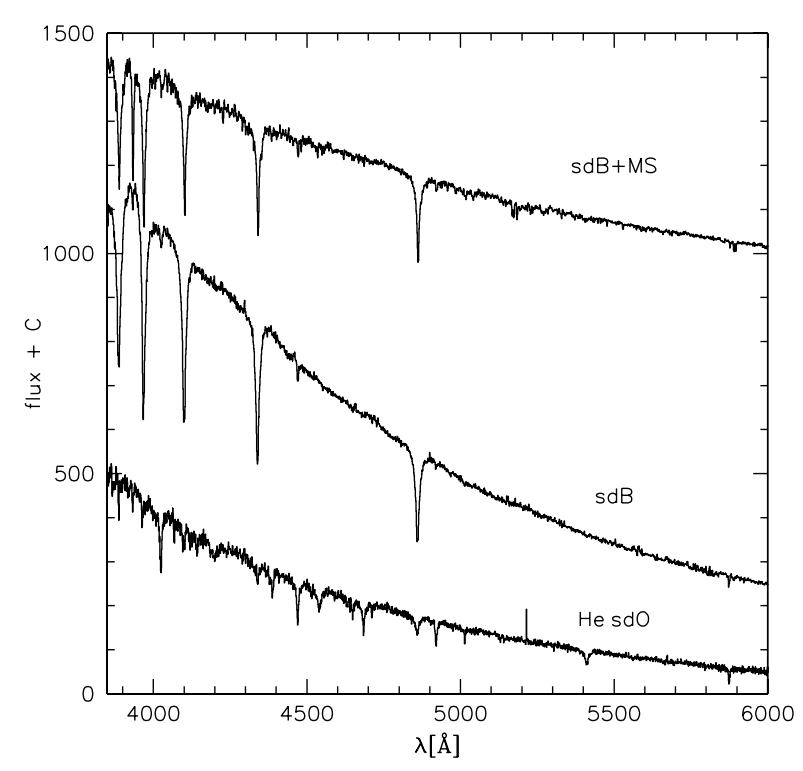

Fig. 3. Flux calibrated SDSS spectra of a single-lined sdB, a helium rich sdO and an sdB with main sequence companion visible in the spectrum. Note the different slopes of the $\mathrm{sdB}$ and the $\mathrm{sdB}+\mathrm{MS}$ spectra.

temperatures ranging from 20000 to $50000 \mathrm{~K}$ as expected for hot subdwarf stars. Different surface gravities, chemical compositions and interstellar reddening are not accounted for but would explain the observed scatter of the stars.

It is interesting to note that there is an obvious lack of blue horizontal branch (BHB) stars with effective temperatures below
$20000 \mathrm{~K}$ compared to the sdBs with higher temperatures. This gap is not a result of selection effects because the BHB stars are brighter than the sdBs at optical wavelengths. We conclude that the number density of BHB stars in the analysed temperature range must be much smaller than that of sdBs. Newell (1973) was the first to report the existence of such a gap in the twocolour diagram of field blue halo stars, which was subsequently also found in some globular clusters (Momany et al. 2004). The reason for this gap remains unclear (see the review by Catelan 2009).

\subsection{High radial velocity sample (HRV)}

The radial velocities of all identified hot subdwarf stars (both single- and double-lined) were measured by fitting a set of mathematical functions (Gaussians, Lorentzians and polynomials) to the hydrogen Balmer lines as well as helium lines, if present, using the FITSB2 routine (Napiwotzki et al. 2004a) and the Spectrum Plotting and Analysis Suite (SPAS) developed by Hirsch. Figure 4 shows the RVs of 1002 hot subdwarf stars.

Most of the known sdB binaries are bright objects $(V \simeq$ 10-14 mag), and the vast majority of them belong to the Galactic disk population (Altmann et al. 2004). Due to the fact that these binary systems are close to the Sun they rotate around the Galactic centre with approximately the same velocity. For this reason, the system velocities of most $\mathrm{sdB}$ binaries are low relative to the Sun. One quarter of the known systems have $|\gamma|<10 \mathrm{~km} \mathrm{~s}^{-1}, 85 \%$ have $|\gamma|<50 \mathrm{~km} \mathrm{~s}^{-1}$ (see Table A.1). In order to filter out normal thin-disk binaries, which in most cases have RV semiamplitudes less than $100 \mathrm{~km} \mathrm{~s}^{-1}$ (see Fig. 1), we excluded sdBs with RVs lower than $\pm 100 \mathrm{~km} \mathrm{~s}^{-1}$.

Typical hot subdwarf stars fainter than $g \simeq 17$ mag have distances exceeding $4 \mathrm{kpc}$ and therefore likely belong to the 
S. Geier et al.: The MUCHFUSS project - searching for hot subdwarf binaries with massive unseen companions

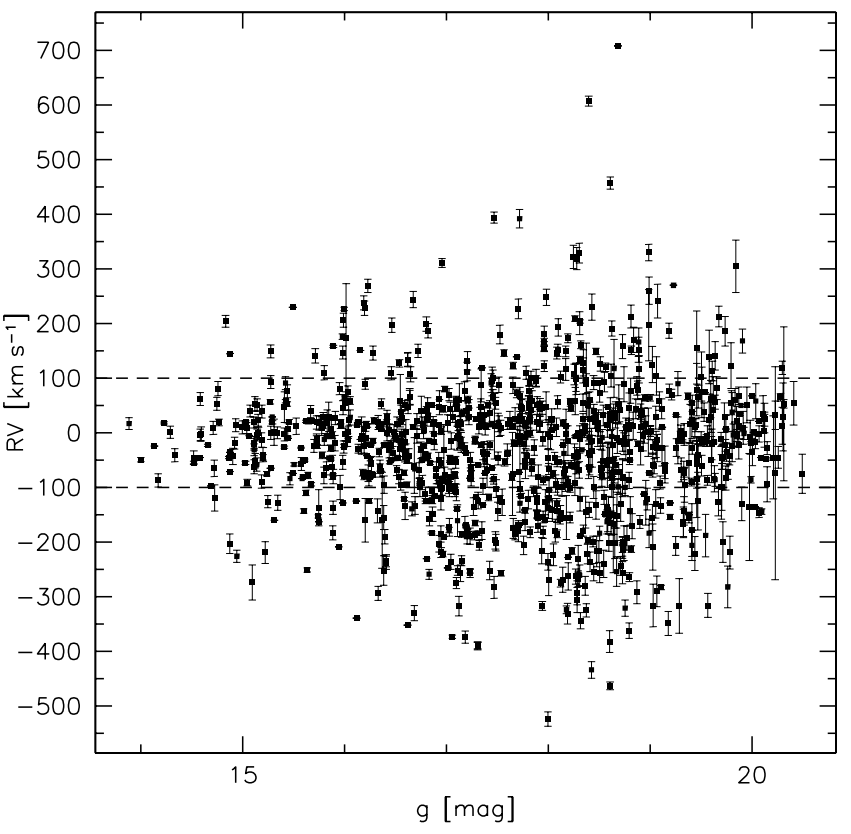

Fig. 4. Heliocentric radial velocities of 1002 subdwarfs plotted against $g$-magnitude. The two dashed lines mark the RV cut of $\pm 100 \mathrm{~km} \mathrm{~s}^{-1}$.

Table 1. Survey observations.

\begin{tabular}{lcc}
\hline \hline Date & Telescope \& Instrument & Observers \\
\hline January-June 2008 & CAHA-3.5 m/TWIN & Service \\
2008/04/29-2008/05/01 & ING-WHT/ISIS & P. M., S. G., \\
& & S. B. \\
2008/08/13-2008/08/17 & CAHA-3.5 m/TWIN & H. H. \\
2008/10/15-2008/10/19 & ESO-NTT/EFOSC2 & A. T. \\
April-July 2008 & ESO-VLT/FORS1 & Service \\
\hline
\end{tabular}

Notes. The first column lists the date of observation, while in the second the used telescope and instrumentation is shown. In the third column the initials of the observers are given.

Galactic halo population. Most of the stars in our sample are fainter than that (see Fig. 4). The velocity distribution in the halo is roughly consistent with a Gaussian of $120 \mathrm{~km} \mathrm{~s}^{-1}$ dispersion (Brown et al. 2005). Figure 5 shows the velocity distributions of our selected objects when separated into bright and faint subsamples. The distribution of the bright subsample $(g<16.5 \mathrm{mag})$ is roughly similar to the one of the faint subsample $(g>16.5 \mathrm{mag})$, the latter extending to more extreme velocities and being somewhat asymmetric. Selecting objects with heliocentric radial velocities exceeding $\pm 100 \mathrm{~km} \mathrm{~s}^{-1}$ we aim to find halo stars with extreme kinematics as well as close binaries with high RV amplitudes.

Another selection criterion is the brightness of the stars. The accuracy of the RV measurements depends on the $S / N$ of the spectra and the existence and strength of the spectral lines. Furthermore, the classification becomes more and more uncertain as soon as the $S / N$ drops below $\simeq 10$ and the probability of including DAs rises. Objects of uncertain type and RV (errors larger than $50 \mathrm{~km} \mathrm{~s}^{-1}$ ) have therefore been excluded. Most of the excluded objects are fainter than $g=19 \mathrm{mag}$. Altogether the target sample consists of 258 stars.

Second epoch medium resolution spectroscopy was obtained starting in 2008 using ESO-VLT/FORS1 $(R \simeq 1800$,

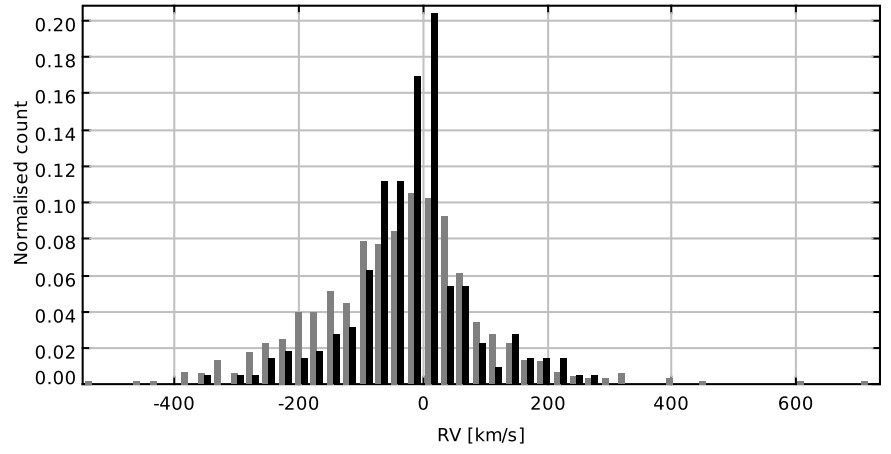

Fig. 5. Radial velocity distribution of the hot subdwarf stars (see Fig. 4). The bright sample $(g<16.5 \mathrm{mag}$, black histogram) contains a mixture of stars from the disk and the halo population. The faint sample ( $g>16.5 \mathrm{mag}$, grey histogram) contains the halo population. The peak in the bright subsample around zero RV is caused by the thin disk population. The asymmetry in the faint subsample where negative RVs are more numerous than positive ones may be due to the presence of large structures in the halo and the movement of the solar system relative to the halo.

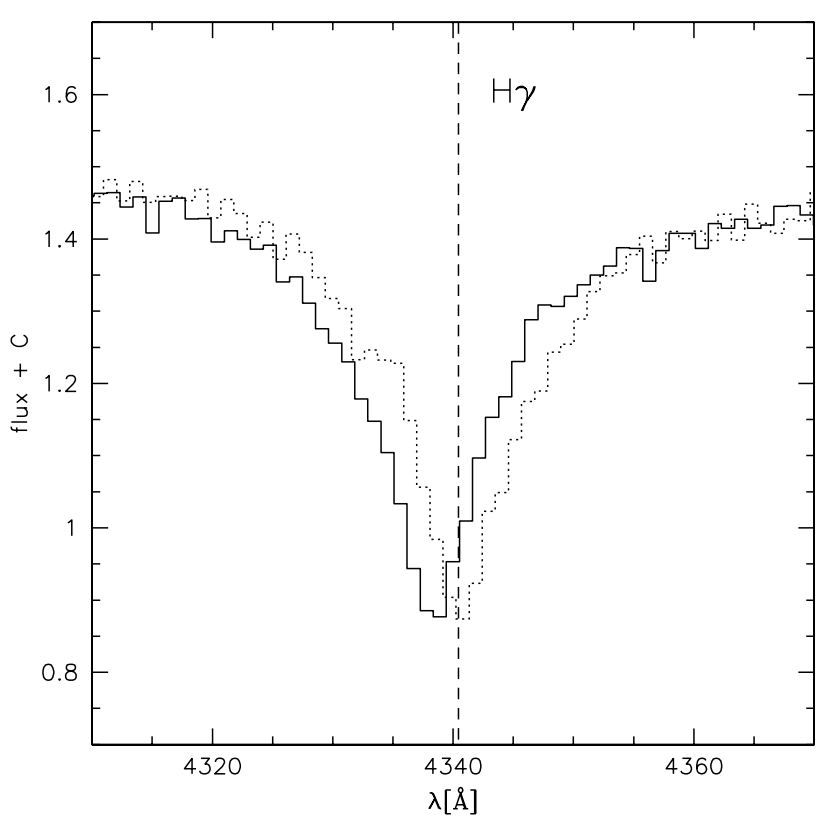

Fig. 6. $\mathrm{H}_{\gamma}$-line of two consecutively taken individual SDSS spectra $(\Delta t=0.056 \mathrm{~d})$ of the sdB binary J113840.68-003531.7. The shift in $\mathrm{RV}\left(\simeq 140 \mathrm{~km} \mathrm{~s}^{-1}\right)$ between the two exposures is clearly visible.

$\lambda=3730-5200 \AA)$, WHT/ISIS $(R \simeq 4000, \lambda=3440-5270 \AA)$, CAHA-3.5m/TWIN $(R \simeq 4000, \lambda=3460-5630 \AA)$ and ESONTT/EFOSC2 $(R \simeq 2200, \lambda=4450-5110 \AA)$. A log of our observations is given in Table 1 . Up to now we have reobserved 88 stars. We discovered $\simeq 30$ halo star candidates with constant high radial velocity (see Tillich et al. 2011) as well as 46 systems with radial velocities that were most likely variable.

\subsection{Rapid radial velocity variable sample (RRV)}

All SDSS spectra are co-added from at least three individual "sub-spectra" with typical exposure times of $15 \mathrm{~min}$. In most cases, the sub-spectra are taken consecutively; however, they may be split occasionally over several nights. 
Several SDSS objects are observed more than once, either because the entire spectroscopic plate is re-observed, or because they are in the overlap area between adjacent spectroscopic plates; up to 30 sub-spectra are available for some objects. Consequently, SDSS spectroscopy can be used to probe for radial velocity variations, a method pioneered by RebassaMansergas et al. (2007) to identify close white dwarf plus mainsequence binaries. We have obtained the sub-spectra for all sdBs brighter than $g=18.5 \mathrm{mag}$ from the SDSS Data Archive Server. The quality of individual spectra of stars fainter than this is not sufficient for our analysis. The object spectra were extracted from the FITS files for the blue and red spectrographs, and merged into a single spectrum using MIDAS. From the inspection of these data, we discovered 81 new candidate sdB binaries with radial velocity variations on short time scales, $\simeq 0.02-0.07 \mathrm{~d}$ (see Fig. 6 for an example).

The individual SDSS spectra are perfectly suited to search for close double degenerate binaries. Ongoing projects like SWARMS (Badenes et al. 2009; Mullally et al. 2009) focus on binaries with white dwarf primaries (see also Kilic et al. 2010; Marsh et al. 2010) and use a similar method.

\subsection{Selecting high mass companions}

Time resolved follow-up spectroscopy with a good phase coverage is needed to determine the orbital solutions of the RV variable systems. In order to select the most promising targets for follow-up, we carried out numerical simulations and estimated the probability for a subdwarf binary with known RV shift to host a massive compact companion. We created a mock sample of sdBs with a close binary fraction of $50 \%$.

We adopted the distribution of orbital periods of all known sdB binaries (see Table A.1) approximated by two Gaussians centered at $0.7 \mathrm{~d}$ (width $0.3 \mathrm{~d}$ ) and $5.0 \mathrm{~d}$ (width $3.0 \mathrm{~d}$ ) and assumed that $82 \%$ of the binaries belong to the short period population. The short period Gaussian was truncated at $0.05 \mathrm{~d}$, which is considered the minimum period for an sdB binary, because the subdwarf primary starts filling its Roche lobe for shorter periods and typical companion masses. Since stable Roche lobe overflow and the accretion onto the companion would dramatically change the spectra of these stars, we can safely presume that our sample does not contain such objects.

The orbital inclination angles are assumed to be randomly distributed, but for geometrical reasons binaries at high inclinations are more likely to be observed than binaries at low inclinations. To account for this, we used the method described in Gray (1992) and adopted a realistic distribution of inclination angles.

We assumend the canonical value of $0.47 M_{\odot}$ for the $\mathrm{sdB}$ masses. The distribution of companion masses was based on the results of Geier et al. (2010b). The distribution of the low mass companions was approximated by a Gaussian centered at $0.4 M_{\odot}$ (width $0.3 M_{\odot}$ ). The fraction of massive compact companions is estimated as $2 \%$ of the close binary population based on binary population synthesis models (Geier et al. 2010b). The mass distribution of these companions was approximated by a Gaussian centered at $2.0 M_{\odot}$ (width $1.0 M_{\odot}$ ).

We adopted a Gaussian distribution for the system velocities with a dispersion of $120 \mathrm{~km} \mathrm{~s}^{-1}$, a typical value for halo stars (Brown et al. 2005). Two RVs were taken from the model RV curves at random times and the RV difference was calculated for each of the $10^{6}$ binaries in the simulation sample. This selection criterion corresponds to the HRV sample. For given RV difference and timespan between the measurements the fraction

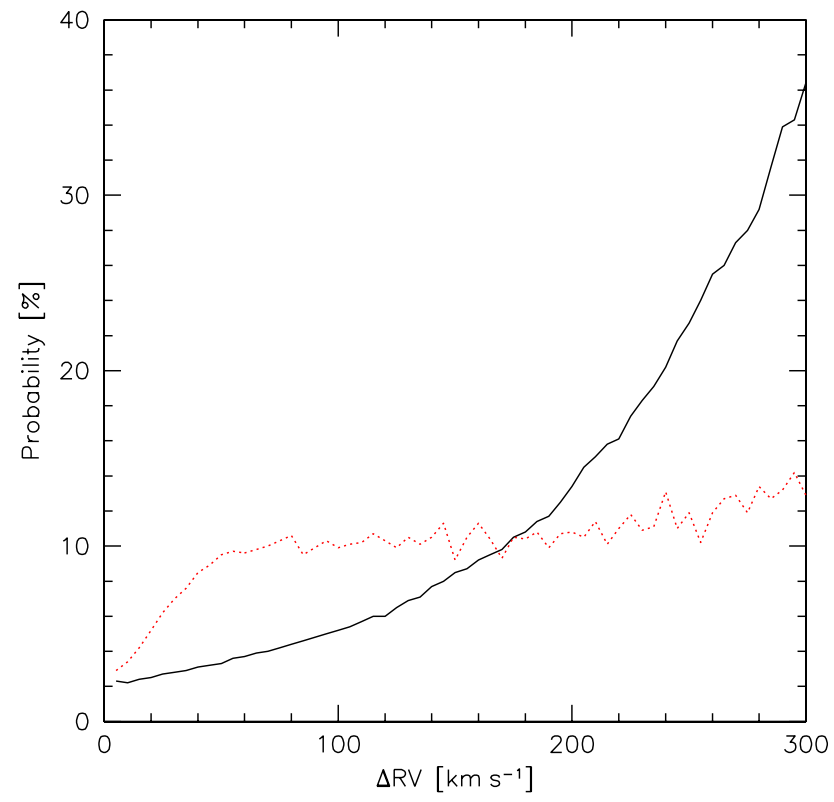

Fig. 7. Probability for an sdB binary to host a massive compact companion and to be seen at sufficiently high inclination to unambiguously identify it from its binary mass function plotted against the RV shift within random times (solid curves, HRV sample) or on short timescales (dotted curve, RRV sample).

of systems with minimum companion masses exceeding $1 M_{\odot}$ was computed.

Figure 7 shows the fraction of massive compact companions with unambiguous mass functions plotted against the RV shift between two measurements taken at random times (solid curve). It is quite obvious that binaries with high RV shifts are more likely to host massive companions. The probability for a high mass companion $\left(>1 M_{\odot}\right)$ at high inclination is raised by a factor of ten as soon as the RV shift exceeds $200 \mathrm{~km} \mathrm{~s}^{-1}$.

In order to check whether the selection of high velocities rather than high velocity shifts has an impact on the probability of finding sdB binaries with massive compact companions we used the same simulation. In Fig. 8 the fraction of these binaries is plotted against only one RV measurement taken at a random time. It can be clearly seen that the detection probability rises significantly for stars with high RVs. Selecting the fastest stars in the halo therefore makes sense when searching for massive compact companions to sdBs.

Since the individual SDSS spectra were taken within short timespans, another simulation was performed corresponding to the RRV sample. The first RV was taken at a random time, but the second one just 0.03 d later. The dotted curve in Fig. 7 illustrates the outcome of this simulation. As soon as the RV shift exceeds $30 \mathrm{~km} \mathrm{~s}^{-1}$ within $0.03 \mathrm{~d}$, the probability that the companion is massive rises to $\simeq 10 \%$. The reason the probability does not increase significantly with increasing RV shift is that the most massive companions in our simulation have maximum $\mathrm{RV}$ shifts as high as $1000 \mathrm{~km} \mathrm{~s}^{-1}$. At the most common periods $(\simeq 0.5 \mathrm{~d})$, the maximum RV shift within $0.03 \mathrm{~d}$ is then of the order of $100 \mathrm{~km} \mathrm{~s}^{-1}$. RV shifts higher than this within comparable time intervals are not physically plausible.

Our simulation provides quantitative estimates based on our current knowledge of the sdB binary populations. We note that these numbers should be considered as rough estimates only. The 


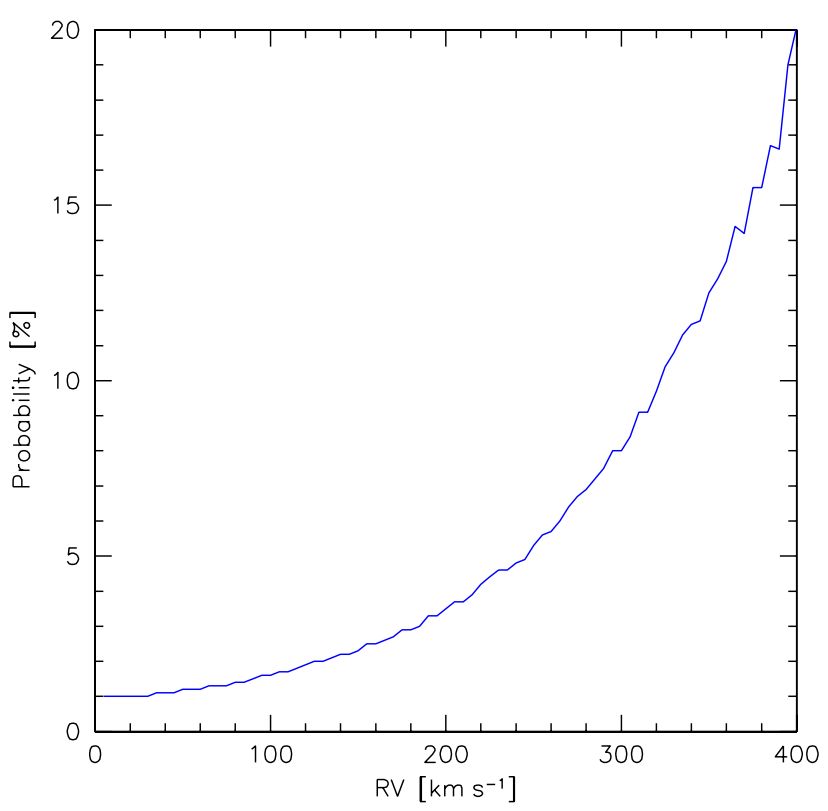

Fig. 8. Same as Fig. 7 except that the probability is plotted against RV at random time.

observed period and companion mass distributions, for example, are highly susceptible to selection effects. The derived numbers are therefore only used to create a priority list and select the best targets for follow-up.

\subsection{Final target sample}

Our sample of promising targets consists of 69 objects in total. 52 stars show significant RV shifts $\left(>30 \mathrm{~km} \mathrm{~s}^{-1}\right)$ within 0.02-0.07 $\mathrm{d}$ and are selected from the RRV sample, while 17 stars show high RV shifts $\left(100-300 \mathrm{~km} \mathrm{~s}^{-1}\right)$ within more than one day and are selected from the HRV sample (see Fig. 9).

In Geier et al. (2011) we showed that the SDSS spectra are well suited to determine atmospheric parameters by fitting synthetic line profiles to the hydrogen Balmer lines $\left(\mathrm{H}_{\beta}\right.$ to $\left.\mathrm{H}_{9}\right)$ as well as He I and He II lines. In order to maximize the quality of the data the single spectra were shifted to rest wavelength and coadded. The quality of the averaged spectra is quite inhomogeneous $(S / N \simeq 20-180$, see Table 2$)$, which affects the accuracy of the parameter determination.

A quantitative spectral analysis was performed in the way described in Lisker et al. (2005) and Ströer et al. (2007). Due to the fact that our sample consists of different subdwarf classes, we used appropriate model grids in each case. For the hydrogenrich and helium-poor $(\log y<-1.0)$ sdBs with effective temperatures below $30000 \mathrm{~K}$ a grid of metal line blanketed LTE atmospheres with solar metallicity was used. Helium-poor sdBs and sdOBs with temperatures ranging from $30000 \mathrm{~K}$ to $40000 \mathrm{~K}$ were analysed using LTE models with enhanced metal line blanketing (O'Toole \& Heber 2006). Metal-free NLTE models (Ströer et al. 2007) were used for hydrogen-rich sdOBs with temperatures below $40000 \mathrm{~K}$ showing moderate He-enrichment $(\log y=-1.0 \ldots 0.0)$ and for hydrogen-rich sdOs. Finally, the HesdOs were analysed with NLTE models taking into account the line-blanketing caused by nitrogen and carbon (Hirsch \& Heber 2009).

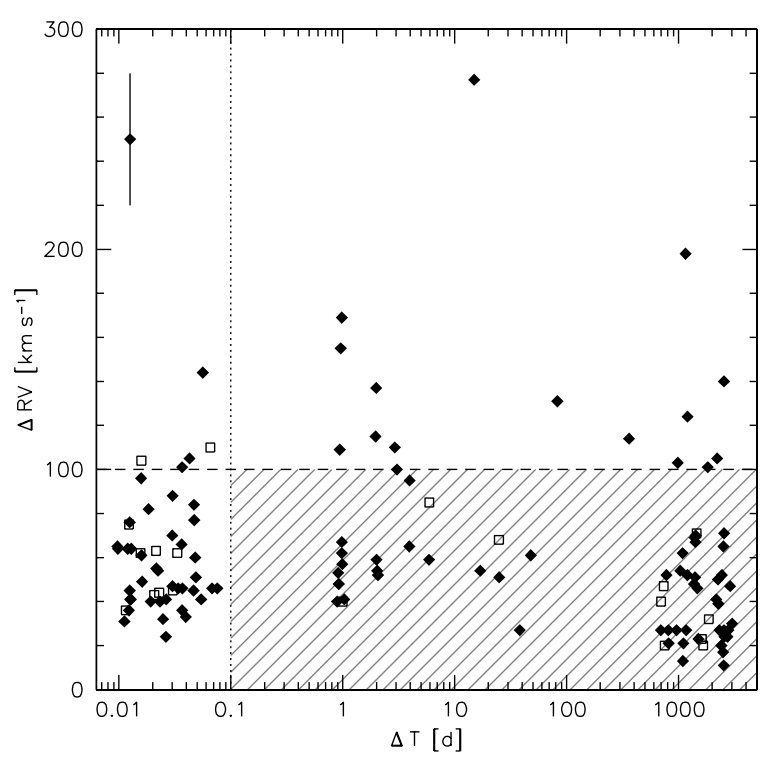

Fig. 9. Highest radial velocity shift between individual spectra plotted against time difference between the corresponding observing epochs. The dashed horizontal line marks the selection criterion $\Delta R V>$ $100 \mathrm{~km} \mathrm{~s}^{-1}$, the dotted vertical line the selection criterion $\Delta T<0.1 \mathrm{~d}$. All objects fulfilling at least one of these criteria lie outside the shaded area and belong to the top candidate list for the follow-up campaign. The filled diamonds mark sdBs, while the blank squares mark He-sdOs.

Spectral lines of hydrogen and helium were fitted by means of chi-squared minimization using SPAS, and statistical errors were calculated with a bootstrapping algorithm. Minimum errors reflecting systematic shifts when using different model grids $\left(\Delta T_{\text {eff }}=500 \mathrm{~K} ; \Delta \log g=0.05 ; \Delta \log y=0.1\right.$, for a discussion see Geier et al. 2007) have been adopted in cases where the statistical errors were lower. Example fits for a typical $\mathrm{sdB}$, an sdOB and a He-sdO star are shown in Fig. 10.

In addition to statistical uncertainities, systematic effects have to be taken into account in particular for sdB stars. The higher Balmer lines $\left(\mathrm{H}_{\epsilon}\right.$ and higher $)$ at the blue end of the spectral range are very sensitive to changes in the atmospheric parameters. However, the SDSS spectral range restricts our analysis to the Balmer lines from $\mathrm{H}_{\beta}$ to $\mathrm{H}_{9}$. In high $S / N$ data these lines are sufficient to measure accurate parameters as has been shown in Geier et al. (2011). In spectra of lower quality the bluest lines $\left(\mathrm{H}_{9}\right.$ and $\left.\mathrm{H}_{8}\right)$ are dominated by noise and cannot be used any more. In order to check whether this leads to systematic shifts in the parameters as reported in Geier et al. (2010b) we made use of the individual SDSS spectra. We chose objects with multiple spectra, which have a $S / N$ comparable to the lowest quality data in our sample $(\simeq 20)$. The atmospheric parameters were obtained from each individual spectrum. Average values of $T_{\text {eff }}$ and $\log g$ were calculated and compared to the atmospheric parameters derived from the analysis of the appropriate coadded spectrum. For effective temperatures ranging from $27000 \mathrm{~K}$ and $39000 \mathrm{~K}$ no significant systematic shifts were found. This means that the error is dominated by statistical noise. However, for temperatures as low as $25000 \mathrm{~K}$ systematic shifts of the order of $-2500 \mathrm{~K}$ in $T_{\text {eff }}$ and -0.35 in $\log g$ are present. For sdBs with low effective temperatures and signal-to-noise, the atmospheric parameters are therefore systematically underestimated. Only three stars in our sample have temperatures in this range. Since their 

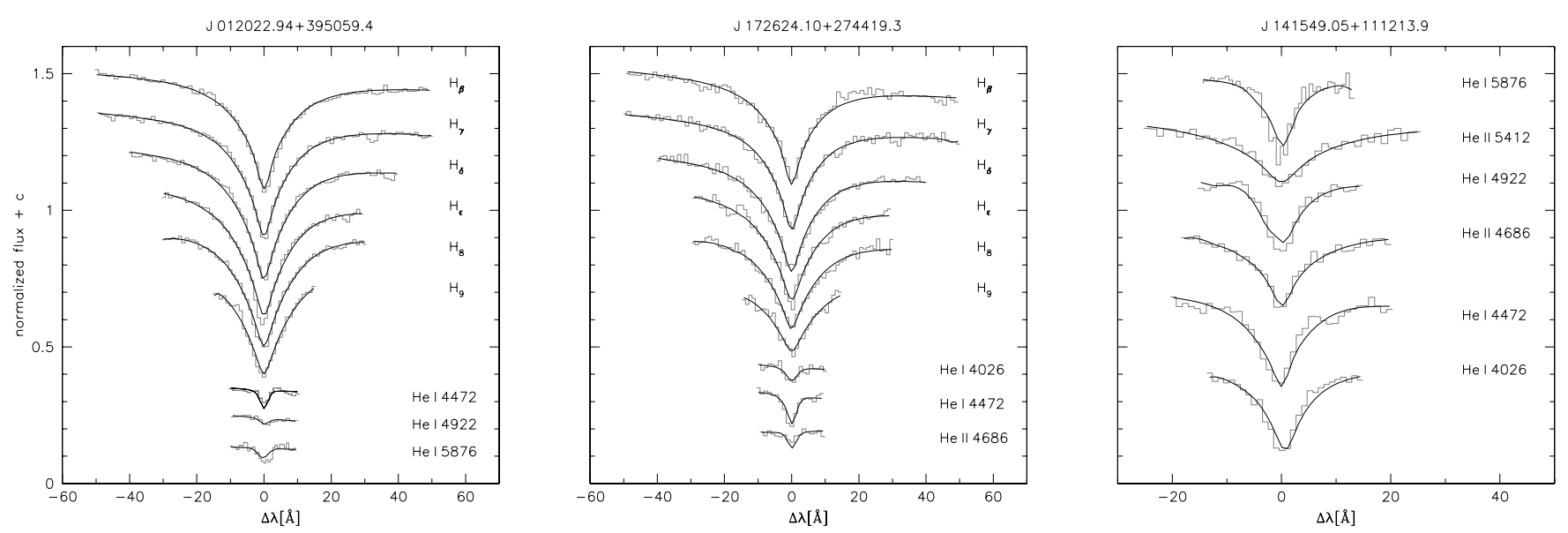

Fig. 10. Example fits of hydrogen and helium lines with model spectra for an sdB (left panel), an sdOB (middle panel) and a He-sdO star (right panel). The atmospheric parameters of these stars are given in Tables 3 and 4.

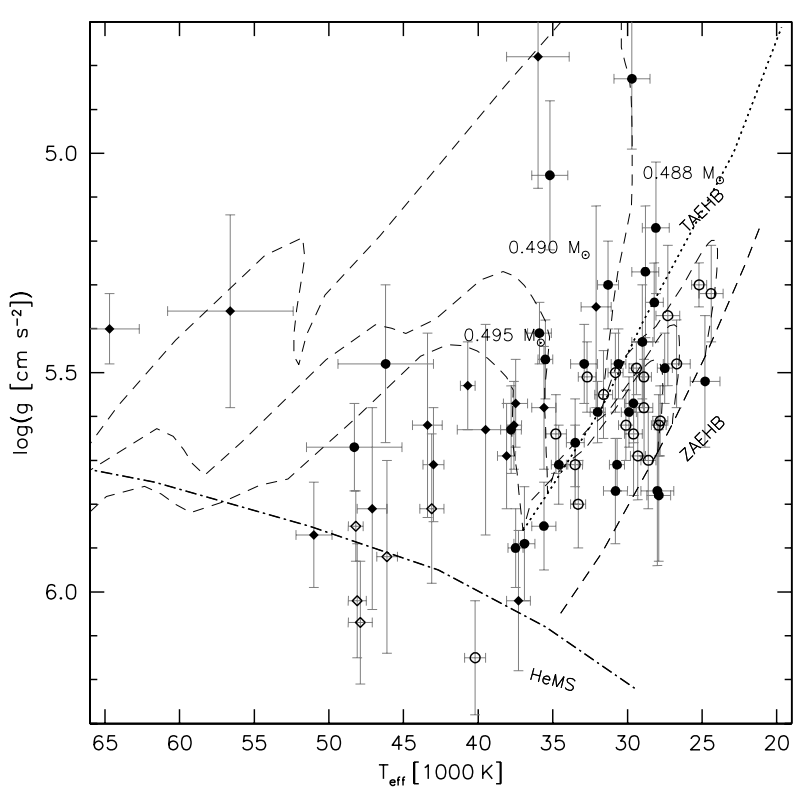

Fig. 11. $T_{\text {eff }}-\log g$ diagram of our target sample. The helium main sequence (HeMS) and the EHB band (limited by the zero-age EHB, ZAEHB, and the terminal-age EHB, TAEHB) are superimposed with EHB evolutionary tracks for subsolar metallicity $(\log z=-1.48)$ from Dorman et al. (1993).

coadded spectra are of reasonable quality $(S / N=34-167)$, systematic shifts should be negligible in these cases. Because all important lines of He I and He II are well covered by the SDSS spectral range, systematic effects should be negligible in the case of He-rich sdO/Bs as well.

The parameters of the sample are given in Tables 3 and 4 . Seven stars have already been analysed in Geier et al. (2011). The sample consists of 38 hydrogen rich sdBs, 13 sdOBs and 3 hydrogen rich sdOs. Thirteen stars are helium rich sdOs (HesdOs) and J134352.14+394008.3 belongs to the rare class of helium rich sdBs.

Our SDSS sample reaches down to fainter magnitudes and hence, larger distances than any previous survey. In an ongoing project Green et al. (2008) analyse all hot subdwarfs from the PG survey down to $\simeq 14.0 \mathrm{mag}$. The sample of hot subdwarf stars analysed in the course of the SPY survey reaches down to $\simeq 16.5$ mag (Lisker et al. 2005; Ströer et al. 2007), quite similar to the sample of sdBs from the Hamburg Quasar Survey analysed by Edelmann et al. (2003).

Spectroscopic distances to our stars have been calculated as described in Ramspeck et al. (2001) assuming the canonical mass of $0.47 M_{\odot}$ for the subdwarfs and using the formula given by Lupton ${ }^{5}$ to convert SDSS-g and $r$ magnitudes to Johnson $V$ magnitudes. Interstellar reddening was once again neglected in these calculations, too. The distances range from $1 \mathrm{kpc}$ to $>16 \mathrm{kpc}$. Since the SDSS footprint is roughly perpendicular to the Galactic disk, these distances tell us something about the population membership of our stars. These subdwarfs most likely belong to the thick disk or the halo with small contributions of thin disk stars.

Figure 11 shows a $T_{\text {eff }}-\log g$ diagram of the top target sample. Most of our stars were born in an environment of low metallicity (thick disk or halo). Dorman et al. (1993) calculated evolutionary tracks for different metallicities of the subdwarf progenitor stars. For lower metallicities, the evolutionary tracks (and with them, the location of the EHB) are shifted towards higher temperatures and lower surface gravities. In Fig. 11 the $T_{\text {eff }}-\log g$ diagram is superimposed with evolutionary tracks and an EHB calculated for a subsolar metallicity of $\log z=-1.48$, which is consistent with a mixture between thick disk and halo population. Evolutionary tracks for solar metallicity are given in Fig. 12 for comparison.

Most of the sdB stars with hydrogen-rich atmospheres are found on or slightly above the EHB band implying an evolutionary status as core helium-burning EHB or shell helium-burning post-EHB stars. The sample contains only three hydrogen rich sdOs, which are thought to be evolved post-EHB stars in a transition state. The He-sdOs cluster near the HeMS at temperatures of $\simeq 45000 \mathrm{~K}$. This is fully consistent with the results from the PG and the SPY surveys (Green et al. 2008; Lisker et al. 2005; Ströer et al. 2007) and illustrates that our sample is not biased (see Fig. 12).

Compared to other studies, we find only a few stars with temperatures lower than $27000 \mathrm{~K}$. Furthermore, the scatter around

\footnotetext{
5 http://www. sdss.org/dr6/algorithms / sdssUBVRITransform. html
} 


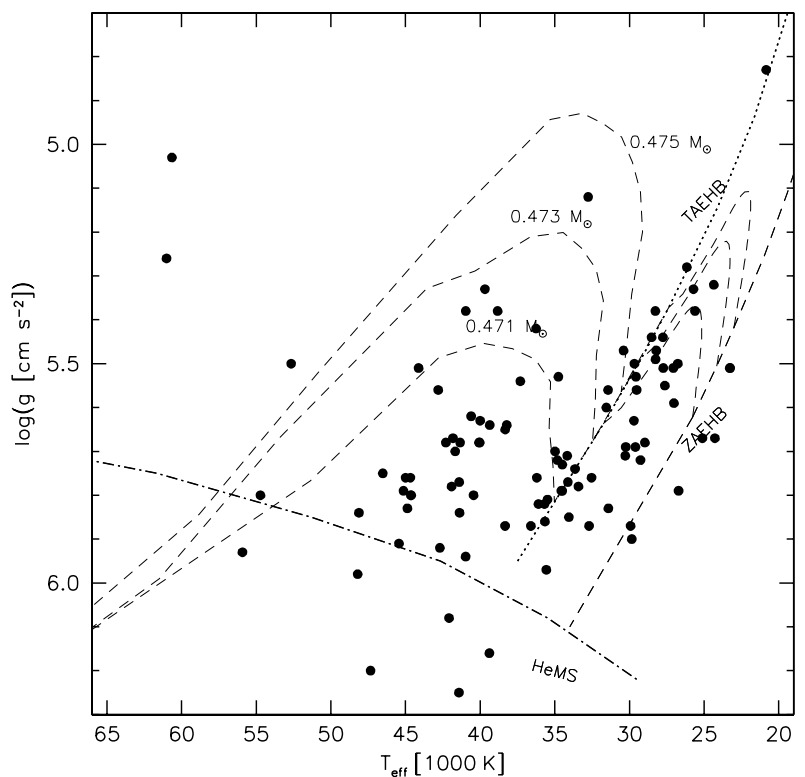

Fig. 12. $T_{\text {eff }}-\log g$ diagram of the hot subdwarfs from the SPY project (Lisker et al. 2005; Ströer et al. 2007). The helium main sequence (HeMS) and the EHB band (limited by the zero-age EHB, ZAEHB, and the terminal-age EHB, TAEHB) are superimposed with EHB evolutionary tracks for solar metallicity from Dorman et al. (1993).

the EHB seems to be systematically shifted towards higher temperatures and lower surface gravities. According to our study of systematic errors in the parameter determination, it is unlikely that this causes the effect. However, higher quality data would be necessary to verify this. Another possible explanation might be related to the volume of the sample. Since hot subdwarfs of lower temperature are brighter in the optical range because of the lower bolometric correction, we may already see all of them in a fixed volume, while the fraction of hot stars is still rising at fainter magnitudes.

In Fig. 13 the helium abundance is plotted against effective temperature. The general correlation of helium abundance with effective temperature and the large scatter in the region of the sdB stars have been observed in previous studies as well. Two sequences of helium abundance among the sdB stars as reported by Edelmann et al. (2003) could not be identified.

One has to keep in mind that our sample consists of RV variable stars only. In Fig. 11 a lack of such stars at the hot end of the EHB is visible. Green et al. (2008) reported similar systematics in their bright PG sample. The reason for this behaviour is not fully understood yet. According to the model of Han et al. (2002, 2003) and Han (2008) sdBs with thin hydrogen envelopes situated at the hot end of the EHB may be formed after the merger of two helium WDs. Since merger remnants are single stars, they are not RV variable.

The top target sample includes $13 \mathrm{He}$-sdOs for which RV shifts of up to $100 \mathrm{~km} \mathrm{~s}^{-1}$ have been detected within short timespans of $0.01-0.1 \mathrm{~d}$. In total $20 \mathrm{He}$-sdOs show signs of RV variability. This fraction was unexpected since the fraction of close binary He-sdOs from the SPY sample turned out to be $4 \%$ at most (Napiwotzki 2008) ${ }^{6}$.

\footnotetext{
${ }^{6}$ Green et al. (2008) suggested that the binary fraction of He-sdO stars may be comparable to the binary fraction of sdBs.
}

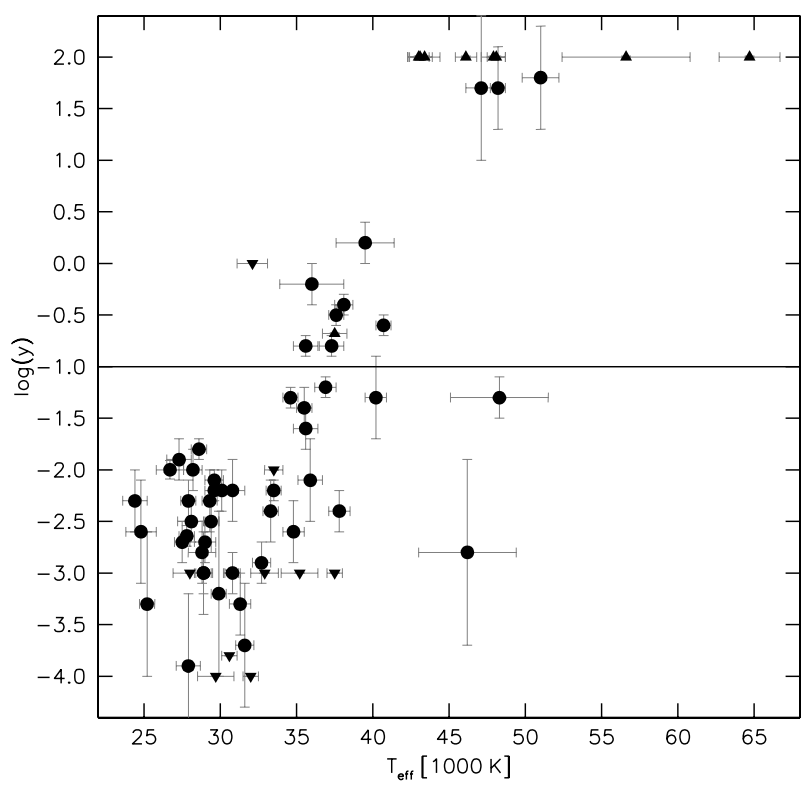

Fig. 13. Helium abundance $\log y$ plotted against effective temperature (see Tables 3,4). The solid horizontal line marks the solar value. Lower and upper limits are marked with upward and downward triangles.

\section{Summary and outlook}

In this paper we introduced the MUCHFUSS project, which aims at finding sdBs in close binaries with massive compact companions. We identified 1100 hot subdwarf stars from the SDSS by colour selection and visual inspection of their spectra. Stars with high absolute radial velocities have been selected to efficiently remove normal sdB binaries from the thin disk population and were reobserved. We have found 46 binary candidates with significant RV shifts. Additionally, 81 stars with RV shifts on short timescales were found from the analysis of individual SDSS spectra.

Targets for follow-up spectroscopy were chosen using numerical simulations based on the properties of the known sdB close binary population and theoretical predictions about the relative fraction of massive compact companions. We selected 69 binaries with high RV shifts as well as significant RV shifts on short timescales as good candidates for massive compact companions and have determined their atmospheric parameters, spectroscopic distances, and population memberships.

The multi-site follow-up campaign started in 2009 and is being conducted with medium resolution spectrographs mounted on several different telescopes, most of which are 4-m class. First results are presented in Geier et al. (2011).

Acknowledgements. A.T., S.G. and H.H. are supported by the Deutsche Forschungsgemeinschaft (DFG) through grants HE1356/45-1, HE1356/49-1, and HE1356/44-1, respectively. R. $\varnothing$. acknowledges funding from the European Research Council under the European Community's Seventh Framework Programme (FP7/2007-2013)/ERC grant agreement No. 227224 (PROSPERITY), as well as from the Research Council of K.U.Leuven grant agreement GOA/2008/04. B.N.B. acknowledges the support of the National Science Foundation, under award AST-0707381.

Funding for the SDSS and SDSS-II has been provided by the Alfred P. Sloan Foundation, the Participating Institutions, the National Science Foundation, the US Department of Energy, the National Aeronautics and Space Administration, the Japanese Monbukagakusho, the Max Planck Society, and the Higher Education Funding Council for England. The SDSS Web Site is http://www . sdss.org/. 
The SDSS is managed by the Astrophysical Research Consortium for the Participating Institutions. The Participating Institutions are the American Museum of Natural History, Astrophysical Institute Potsdam, University of Basel, University of Cambridge, Case Western Reserve University, University of Chicago, Drexel University, Fermilab, the Institute for Advanced Study, the Japan Participation Group, Johns Hopkins University, the Joint Institute for Nuclear Astrophysics, the Kavli Institute for Particle Astrophysics and Cosmology, the Korean Scientist Group, the Chinese Academy of Sciences (LAMOST), Los Alamos National Laboratory, the Max-Planck-Institute for Astronomy (MPIA), the Max-Planck-Institute for Astrophysics (MPA), New Mexico State University, Ohio State University, University of Pittsburgh, University of Portsmouth, Princeton University, the United States Naval Observatory, and the University of Washington.

\section{References}

Abazajian, K., Adelman-McCarthy, J. K., Agüeros, M. A., et al. 2009, ApJS, 182,543

Ahmad, A., Jeffery, C. S., \& Fullerton, A. W. 2004, A\&A, 418, 275

Altmann, M., Edelmann, H., \& de Boer, K. S. 2004, A\&A, 414, 181

Badenes, C., Mullally, F., Thompson, S. E., \& Lupton, R. H. 2009, ApJ, 707, 971

Bloemen, S., Marsh, T. R., Østensen, R. H., et al. 2011, MNRAS, 410, 1787

Brown, W. R., Geller, M. J., Kenyon, S. J., et al. 2005, AJ, 130, 109

Castelli, F., \& Kurucz, R. L. 2003, IAU Symp., 210, 20

Catelan, M. 2009, Ap\&SS, 320, 261

Dorman, B., Rood, R. T., \& O’Connell, R. W. 1993, ApJ, 419, 596

Drechsel, H., Heber, U., Napiwotzki, R., et al. 2001, A\&A, 379, 893

Edelmann, H. 2008, ASP Conf. Ser., 392, 187

Edelmann, H., Heber, U., Hagen, H.-J., et al. 2003, A\&A, 400, 939

Edelmann, H., Heber, U., Lisker, T., \& Green, E. M. 2004, Ap\&SS, 291, 315

Edelmann, H., Heber, U., Altmann, M., Karl, C., \& Lisker, T. 2005, A\&A, 442, 1023

Eisenstein, D. J., Liebert, J., Harris, H. C., et al. 2006, ApJS, 167, 40

For., B.-Q., Green, E. M., O’Donoghue, D., et al. 2006, ApJ, 642, 1117

For, B.-Q., Edelmann, H., Green, E. M., et al. 2008, ASP Conf. Ser., 392, 203

For, B.-Q., Green, E. M., Fontaine, G., et al. 2010, ApJ, 708, 253

Geier, S., Nesslinger, S., Heber, U., et al. 2007, A\&A, 464, 299

Geier, S., Nesslinger, S., Heber, U., et al. 2008, A\&A, 477, L13

Geier, S., Heber, U., Kupfer, T., \& Napiwotzki, R. 2010a, A\&A, 515, A37

Geier, S., Heber, U., Podsiadlowski, Ph., et al. 2010b, A\&A, 519, A25

Geier, S., Maxted, P. F. L., Napiwotzki, R., et al., 2011, A\&A, 526, A39

Gray, D. F. 1992, The observation and analysis of stellar photospheres, 2nd edn.

(Cambridge: University Press)

Green, E. M., Fontaine, G., Hyde, E. A., For, B.-Q., \& Chayer, P. 2008, ASP Conf. Ser., 392, 75

Green, R. F., Schmidt, M., \& Liebert, J. 1986, ApJS, 61, 305

Green, E. M., For, B., Hyde, E. A., et al. 2004, Ap\&SS, 291, 267

Green, E. M., For, B., Hyde, E. A., et al. 2005, ASP Conf. Ser., 334, 363

Han, Z. 2008, A\&A, 484, L31

Han Z., Podsiadlowski P., Maxted P. F. L., Marsh T. R., \& Ivanova N. 2002, MNRAS, 336, 449

Han, Z., Podsiadlowski, P., Maxted, P. F. L., \& Marsh, T. R. 2003, MNRAS, 341 , 669

Heber, U. 1986, A\&A, 155, 33

Heber, U. 2009, ARA\&A, 47, 211

Heber, U., Drechsel, H., Østensen, R., et al. 2004, A\&A, 420, 251

Hirsch, H., \& Heber, U. 2009, JPhCS, 172, 2015

Iben, I., \& Tutukov, A. V. 1984, ApJ, 284, 719

Jester, S., Schneider, D. P., Richards, G. T., et al. 2005, AJ, 130, 873

Karl, C., Heber, U., Napiwotzki, R., \& Geier, S. 2006, Baltic Astron., 15, 151

Kawka, A., Vennes, S., Németh, P., Kraus, M., \& Kubát, J. 2010, MNRAS, 408, 992

Kilic, M., Brown, W. E., Allende Prieto, C., \& Kenyon, S. J. 2010, ApJ, 716, 122 Kleinman, S. J. 2010, AIP Conf. Ser., 1237, 156
Koen, C. 2009, MNRAS, 393, 1370

Koen, C., Kilkenny, D., O’Donoghue, D., van Wyk, F., \& Stobie, R. S. 1997, MNRAS, 285, 645

Koen, C., Kilkenny, D., Pretorius, M. L., \& Frew, D. J. 2010, MNRAS, 401, 1850

Leibundgut, B. 2001, ARA\&A, 39, 67

Lisker, T., Heber, U., Napiwotzki, R., et al. 2005, A\&A, 430, 223

Marsh, T. R., Gaensicke, B. T., Steeghs, D., et al. 2010, ApJL, submitted [arXiv: 1002.4677]

Maxted, P. F. L., Marsh, T. R., \& North, R. C. 2000a, MNRAS, 317, L41

Maxted, P. F. L., Moran, C. K. J., Marsh, T. R., \& Gatti, A. A. 2000b, MNRAS, 311,877

Maxted, P. F. L., Heber, U., Marsh, T. R., \& North, R. C. 2001, MNRAS, 326, 139

Maxted, P. F. L., Marsh, T. R., Heber, U., et al. 2002, MNRAS, 333, 231

Maxted, P. F. L., Morales-Rueda, L., \& Marsh, T. 2004, Ap\&SS, 291, 307

Mereghetti, S., Tiengo, A., Esposito, P., et al. 2009, Science, 325, 1222

Momany, Y., Bedin, L. R., Cassisi, S., et al. 2004, A\&A, 420, 605

Morales-Rueda, L., Maxted, P. F. L., Marsh, T. R., North, R. C., \& Heber, U. 2003a, MNRAS, 338, 752

Morales-Rueda, L., Marsh, T. R., North, R. C., \& Maxted, P. F. L. 2003b, in White Dwarfs, ed. D. de Martino, R. Silvotti, J.-E. Solheim, \& R. Kalytis, 57

Morales-Rueda, L., Maxted, P. F. L., Marsh, T. R., Kilkenny, D., \& O’Donoghue, D. 2005, ASP Conf. Ser., 334, 333

Morales-Rueda, L., Maxted, P. F. L., Marsh, T. R., Kilkenny, D., \& O’Donoghue, D. 2006, Baltic Astron., 15, 187

Moran, C., Maxted, P. F. L., Marsh, T. R., Saffer, R. A., \& Livio, M. 1999, MNRAS, 304, 535

Müller, S., Geier, S., \& Heber, U. 2010, unpublished [arXiv: 1012. 3908]

Mullally, F., Badenes, C., Thompson, S. E., \& Lupton, R. 2009, ApJ, 707, 51

Napiwotzki, R. 2008, ASP Conf. Ser., 392, 139

Napiwotzki, R., Edelmann, H., Heber, U., et al. 2001, A\&A, 378, L17

Napiwotzki, R., Karl, C., Lisker, T., et al. 2004a, Ap\&SS, 291, 321

Napiwotzki, R., Yungelson, L., Nelemans, G., et al. 2004b, ASP Conf. Ser., 318, 402

Nelemans, G. 2010, Ap\&SS, 329, 25

Newell, E. B. 1973, ApJS, 26, 37

Østensen, R., Oreiro, R., Drechsel, H., et al. 2007, ASP Conf. Ser., 372, 483

Østensen, R., Green, E. M., Bloemen, S., et al. 2010, MNRAS, 408, 51

Orosz, J. A., \& Wade, R. A. 1999, MNRAS, 310, 773

O'Toole, S. J., Heber, U., \& Benjamin, R. A. 2004, A\&A, 422, 1053

O’Toole, S. J., Napiwotzki, R., Heber, U., et al. 2006, Baltic Astron., 15, 61

Perets, H. B., Gal-Yam, A., Mazzali, P. A., et al. 2010, Nature, 465, 322

Perlmutter, S., Aldering, G., Goldhaber, G., et al. 1999, ApJ, 517, 565

Pfahl, E., Rappaport, S., \& Podsiadlowski, Ph. 2003, ApJ, 597, 1036

Podsiadlowski, Ph., Rappaport, S., \& Pfahl, E. D. 2002, ApJ, 565, 1107

Polubek, G., Pigulski, A., Baran, A., \& Udalski, A. 2007, ASP Conf. Ser., 372, 487

Ramspeck, M., Heber, U., \& Edelmann, H. 2001, A\&A, 379, 235

Rebassa-Mansergas, A., Gänsicke, B. T., Rodrǵuez-Gil., P., Schreiber, M. R., \& Koester, D. 2007, MNRAS, 382, 1377

Reed, M., Terndrup, D. N., Østensen, R. H., et al. 2010, Ap\&SS, 329, 83

Riess, A. G., Fillipenko, A. V., Challis, P., et al. 1998, AJ, 116, 1009

Ritter, H., \& Kolb, U. 2009, VizieR Online Data Catalog, 1, 2018

Shimanskii, V. V., Bikmaev, I. F., Borisov, N. V., et al. 2008, ARep, 52, 729

Ströer, A., Heber, U., Lisker, T., et al. 2007, A\&A, 462, 269

Tillich, A., Heber, U., Geier, S., et al. 2011, A\&A, 527, A137

Vučković, M., Aerts, C., Østensen, R., et al. 2007, A\&A, 471, 605

Vučković, M., Østensen, R., Bloemen, S., Decoster, I., \& Aerts, C. 2008, ASP Conf. Ser., 392, 199

Webbink, R. F. 1984, ApJ, 277, 355

Wils, P., di Scala, G., \& Otero, S. 2007, IBVS, 5800, 1

York, D. G., Adelman, J., Anderson, J. E., et al. 2000, AJ, 120, 1579

Yungelson, L. R., \& Tutukov, A. V. 2005, ARep, 49, 871

Wang, B., Meng, X., Chen, X., \& Han, Z. 2009, MNRAS, 395, 847 
Table 2. Priority targets for follow-up.

\begin{tabular}{|c|c|c|c|c|c|c|c|c|c|}
\hline Object & & $g$ & No. & $S / N$ & Object & & $g$ & No. & $S / N$ \\
\hline J002323.99-002953.2 & PB 5916 & 15.3 & 16 & 116 & $\mathrm{~J} 150513.52+110836.6$ & PG $1502+113$ & 15.1 & 4 & 90 \\
\hline J012022.94+395059.4 & FBS $0117+396$ & 15.2 & 8 & 100 & $\mathrm{~J} 150829.02+494050.9$ & & 17.3 & 3 & 50 \\
\hline J012739.35+404357.8 & & 16.5 & 8 & 59 & $\mathrm{~J} 151415.66-012925.2$ & & 16.8 & 5 & 48 \\
\hline J052544.93+630726.0 & & 17.6 & 3 & 35 & $\mathrm{~J} 152222.15-013018.3$ & & 17.7 & 5 & 28 \\
\hline $\mathrm{J} 074534.16+372718.5$ & & 17.6 & 5 & 26 & $\mathrm{~J} 152705.03+110843.9$ & & 17.1 & 5 & 39 \\
\hline J075937.15+541022.2 & & 17.5 & 3 & 27 & $\mathrm{~J} 153411.10+543345.2$ & WD 1532+547 & 16.7 & 8 & 52 \\
\hline J082053.53+000843.4 & & 14.9 & 6 & 103 & $\mathrm{~J} 155628.34+011335.0$ & & 16.0 & 8 & 92 \\
\hline J083006.17+475150.4 & & 15.8 & 5 & 95 & J161140.50+201857.0 & & 18.2 & 5 & 20 \\
\hline J085727.65+424215.4 & US 1993 & 18.3 & 4 & 21 & J161817.65+120159.6 & & 17.8 & 4 & 18 \\
\hline J092520.70+470330.6 & & 17.4 & 3 & 33 & $\mathrm{~J} 162256.66+473051.1$ & & 16.0 & 4 & 72 \\
\hline J094856.95+334151.0 & KUV 09460+3356 & 17.4 & 3 & 46 & J163702.78-011351.7 & & 17.1 & 12 & 46 \\
\hline J095229.62+301553.6 & & 18.2 & 3 & 20 & $\mathrm{~J} 164326.04+330113.1$ & PG $1641+331$ & 16.1 & 3 & 55 \\
\hline J095238.93+625818.9 & & 14.5 & 4 & 113 & $\mathrm{~J} 165404.26+303701.8$ & PG $1652+307$ & 15.1 & 4 & 167 \\
\hline $\mathrm{J} 100535.76+223952.1$ & & 18.1 & 4 & 28 & $\mathrm{~J} 170645.57+243208.6$ & & 17.5 & 3 & 39 \\
\hline $\mathrm{J} 102151.64+301011.9$ & & 18.0 & 12 & 34 & $\mathrm{~J} 170810.97+244341.6$ & & 18.2 & 3 & 16 \\
\hline $\mathrm{J} 103549.68+092551.9$ & & 16.0 & 3 & 59 & $\mathrm{~J} 171617.33+553446.7$ & SBSS $1715+556$ & 16.9 & 8 & 39 \\
\hline J110215.97+521858.1 & & 17.2 & 3 & 44 & $\mathrm{~J} 171629.92+575121.2$ & & 17.9 & 4 & 21 \\
\hline $\mathrm{J} 110445.01+092530.9$ & & 16.0 & 4 & 40 & J172624.10+274419.3 & PG 1724+278 & 15.7 & 4 & 107 \\
\hline $\mathrm{J} 112242.69+613758.5$ & PG $1119+619$ & 15.1 & 3 & 87 & $\mathrm{~J} 174516.32+244348.3$ & & 17.4 & 3 & 22 \\
\hline $\mathrm{J} 112414.45+402637.1$ & & 17.7 & 3 & 21 & $\mathrm{~J} 175125.67+255003.5$ & & 17.2 & 4 & 50 \\
\hline $\mathrm{J} 113303.70+290223.0$ & & 17.4 & 3 & 34 & J202313.83+131254.9 & & 17.0 & 3 & 33 \\
\hline $\mathrm{J} 113418.00+015322.1$ & LBQS 1131+0209 & 17.7 & 6 & 30 & J202758.63+773924.5 & & 17.7 & 3 & 22 \\
\hline J113840.68-003531.7 & PG 1136-003 & 14.2 & 10 & 174 & J204300.90+002145.0 & & 17.6 & 9 & 50 \\
\hline $\mathrm{J} 113935.45+614953.9$ & FBS $1136+621$ & 16.8 & 3 & 34 & J204448.63+153638.8 & & 17.7 & 7 & 50 \\
\hline $\mathrm{J} 115358.81+353929.0$ & FBS $1151+359$ & 16.3 & 3 & 48 & J204546.81-054355.6 & & 17.8 & 4 & 29 \\
\hline $\mathrm{J} 115716.37+612410.7$ & FBS $1154+617$ & 16.9 & 5 & 34 & J204613.40-045418.7 & & 16.0 & 3 & 120 \\
\hline $\mathrm{J} 125702.30+435245.8$ & & 17.9 & 3 & 18 & J204940.85+165003.6 & & 17.7 & 7 & 35 \\
\hline $\mathrm{J} 130059.20+005711.7$ & PG $1258+012$ & 16.3 & 3 & 47 & $\mathrm{~J} 210454.89+110645.5$ & & 17.2 & 4 & 37 \\
\hline $\mathrm{J} 130439.57+312904.8$ & LB 28 & 16.8 & 3 & 42 & J211651.96+003328.5 & & 17.7 & 3 & 19 \\
\hline $\mathrm{J} 133638.81+111949.4$ & & 17.0 & 3 & 32 & $\mathrm{~J} 215648.71+003620.7$ & PB 5010 & 17.7 & 3 & 22 \\
\hline $\mathrm{J} 134352.14+394008.3$ & & 18.1 & 3 & 19 & J225638.34+065651.1 & PG 2254+067 & 15.1 & 3 & 86 \\
\hline $\mathrm{J} 135807.96+261215.5$ & & 17.7 & 4 & 23 & J232757.46+483755.2 & & 15.6 & 3 & 92 \\
\hline $\mathrm{J} 140545.25+014419.0$ & PG 1403+019 & 15.6 & 3 & 81 & $\mathrm{~J} 233406.11+462249.3$ & & 17.4 & 3 & 35 \\
\hline J141549.05+111213.9 & & 15.8 & 3 & 82 & $\mathrm{~J} 234528.85+393505.2$ & & 17.3 & 3 & 37 \\
\hline $\mathrm{J} 143153.05-002824.3$ & LBQS 1429-0015 & 17.8 & 8 & 34 & & & & & \\
\hline
\end{tabular}

Notes. Besides the names, the $g$ magnitudes, the number of individual spectra and the $S / N$ of the coadded spectra at $\simeq 4100 \AA$ are given.

Table 3. Priority targets for follow-up (HRV subsample).

\begin{tabular}{|c|c|c|c|c|c|c|c|}
\hline Object & Class & $\begin{array}{l}T_{\text {eff }} \\
{[\mathrm{K}]}\end{array}$ & $\log g$ & $\log y$ & $\begin{array}{c}d \\
{[\mathrm{kpc}]}\end{array}$ & $\begin{array}{c}\Delta R V \\
{\left[\mathrm{~km} \mathrm{~s}^{-1}\right]}\end{array}$ & $\begin{array}{l}\Delta t \\
{[\mathrm{~d}]}\end{array}$ \\
\hline J102151.64+301011.9 & $\operatorname{sdB}$ & $30700 \pm 500$ & $5.71 \pm 0.06$ & $<-3.0$ & $5.8_{-0.5}^{+0.5}$ & $277 \pm 51$ & 14.936 \\
\hline $\mathrm{J} 150829.02+494050.9$ & $\mathrm{sdB}$ & $28200 \pm 600$ & $5.34 \pm 0.09$ & $-2.0 \pm 0.2$ & $6.4_{-0.7}^{+0.8}$ & $211 \pm 18$ & 2161.429 \\
\hline J095229.62+301553.6 & $\mathrm{sdB}$ & $35200 \pm 1200$ & $5.05 \pm 0.17$ & $<-3.0$ & $16.0_{-3.3}^{+3.8}$ & $198 \pm 40$ & 1155.766 \\
\hline $\mathrm{J} 113840.68-003531.7 \dagger$ & $\mathrm{sdB}$ & $30800 \pm 500$ & $5.50 \pm 0.09$ & $-3.0 \pm 0.2$ & $1.3_{-0.2}^{+0.3}$ & $182 \pm 12$ & 0.973 \\
\hline $\mathrm{J} 165404.26+303701.8 \dagger$ & $\operatorname{sdB}$ & $24400 \pm 800$ & $5.32 \pm 0.11$ & $-2.3 \pm 0.3$ & $1.9_{-0.3}^{+0.1}$ & $181 \pm 9$ & 1795.144 \\
\hline $\mathrm{J} 152222.15-013018.3$ & $\mathrm{sdB}$ & $24800 \pm 1000$ & $5.52 \pm 0.15$ & $-2.6 \pm 0.5$ & $4.8_{-0.9}^{-1.3}$ & $173 \pm 36$ & 3.001 \\
\hline $\mathrm{J} 150513.52+110836.6 \dagger$ & $\mathrm{sdB}$ & $33300 \pm 500$ & $5.80 \pm 0.10$ & $-2.4 \pm 0.3$ & $1.5_{-0.2}^{-0.2}$ & $154 \pm 12$ & 0.957 \\
\hline J002323.99-002953.2† & $\operatorname{sdB}$ & $30100 \pm 500$ & $5.62 \pm 0.08$ & $-2.2 \pm 0.2$ & $1.8_{-0.2}^{+0.2}$ & $130 \pm 14$ & 82.784 \\
\hline $\mathrm{J} 202313.83+131254.9$ & $\mathrm{sdB}$ & $29600 \pm 600$ & $5.64 \pm 0.14$ & $-2.1 \pm 0.1$ & $3.8_{-0.6}^{+0.2}$ & $124 \pm 21$ & 1202.795 \\
\hline J012022.94+395059.4 & $\operatorname{sdB}$ & $28900 \pm 500$ & $5.51 \pm 0.08$ & $-3.0 \pm 0.4$ & $1.9_{-0.2}^{+0.0}$ & $114 \pm 11$ & 360.973 \\
\hline $\mathrm{J} 202758.63+773924.5$ & $\mathrm{sdO}$ & $46200 \pm 3200$ & $5.48 \pm 0.18$ & $-2.8 \pm 0.9$ & $8.2_{-1 .}^{-2.2}$ & $114 \pm 48$ & 1.960 \\
\hline J095238.93+625818.9 & $\mathrm{sdB}$ & $27800 \pm 500$ & $5.61 \pm 0.08$ & $-2.64 \pm 0.1$ & $1.2_{-0.1}^{+0.0}$ & $111 \pm 10$ & 2.918 \\
\hline $\mathrm{J} 161140.50+201857.0$ & sdOB & $36900 \pm 700$ & $5.89 \pm 0.13$ & $-1.2 \pm 0.1$ & $6.1_{-0.9}^{+1.1}$ & $108 \pm 36$ & 0.947 \\
\hline $\mathrm{J} 164326.04+330113.1$ & $\mathrm{sdB}$ & $27900 \pm 500$ & $5.62 \pm 0.07$ & $-2.3 \pm 0.2$ & $2.4_{-0.2}^{+0.9}$ & $108 \pm 11$ & 1.990 \\
\hline $\mathrm{J} 204448.63+153638.8$ & $\mathrm{sdB}$ & $29600 \pm 600$ & $5.57 \pm 0.09$ & $-2.2 \pm 0.1$ & $5.7_{-0.7}^{-0.2}$ & $101 \pm 19$ & 3.049 \\
\hline J083006.17+475150.4 & $\mathrm{sdB}$ & $25200 \pm 500$ & $5.30 \pm 0.05$ & $-3.3 \pm 0.7$ & $2.8_{-0.2}^{+0.1}$ & $95 \pm 14$ & 3.961 \\
\hline $\mathrm{J} 204940.85+165003.6$ & He-sdO & $43000 \pm 700$ & $5.71 \pm 0.13$ & $>+2.0$ & $6.2_{-0.9}^{+1.1}$ & $85 \pm 19$ & 5.932 \\
\hline
\end{tabular}

Notes. $\uparrow$ The binary system has been analysed in Geier et al. (2011). 
Table 4. Priority targets for follow-up (RRV subsample).

\begin{tabular}{|c|c|c|c|c|c|c|c|}
\hline Object & Class & $\begin{array}{l}T_{\text {eff }} \\
{[\mathrm{K}]}\end{array}$ & $\log g$ & $\log y$ & $\begin{array}{c}d \\
{[\mathrm{kpc}]}\end{array}$ & $\begin{array}{c}\Delta R V \\
{\left[\mathrm{~km} \mathrm{~s}^{-1}\right]}\end{array}$ & $\begin{array}{l}\Delta t \\
{[\mathrm{~d}]}\end{array}$ \\
\hline J085727.65+424215.4 & $\mathrm{He}-\mathrm{sdO}$ & $39500 \pm 1900$ & $5.63 \pm 0.24$ & $+0.2 \pm 0.2$ & $8.7_{-2.2}^{+3.0}$ & $111 \pm 46$ & 0.066 \\
\hline $\mathrm{J} 161817.65+120159.6$ & $\mathrm{sdB}$ & $32100 \pm 1000$ & $5.35 \pm 0.23$ & - & $8.1_{-2.1}^{+2.2}$ & $105 \pm 31$ & 0.043 \\
\hline $\mathrm{J} 232757.46+483755.2$ & He-sdO & $64700 \pm 2000$ & $5.40 \pm 0.08$ & $>+2.0$ & $4.2_{-0.4}^{+0.1}$ & $105 \pm 24$ & 0.016 \\
\hline $\mathrm{J} 162256.66+473051.1$ & $\mathrm{sdB}$ & $28600 \pm 500$ & $5.70 \pm 0.11$ & $-1.81 \pm 0.1$ & $2.2_{-0.3}^{+0.4}$ & $101 \pm 15$ & 0.037 \\
\hline $\mathrm{J} 163702.78-011351.7$ & He-sdO & $46100 \pm 700$ & $5.92 \pm 0.22$ & $>+2.0$ & $3.8_{-0.9}^{+1.1}$ & $101 \pm 55$ & 0.085 \\
\hline $\mathrm{J} 113303.70+290223.0$ & $\mathrm{sdB} / \mathrm{DA}$ & - & - & - & - & $95 \pm 35$ & 0.016 \\
\hline $\mathrm{J} 135807.96+261215.5$ & $\mathrm{sdB}$ & $33500 \pm 600$ & $5.66 \pm 0.10$ & $<-2.0$ & $5.8_{-0.7}^{+0.8}$ & $87 \pm 29$ & 0.030 \\
\hline $\mathrm{J} 112242.69+613758.5$ & $\mathrm{sdB}$ & $29300 \pm 500$ & $5.69 \pm 0.10$ & $-2.3 \pm 0.3$ & $1.5_{-0.2}^{+0.1}$ & $83 \pm 20$ & 0.047 \\
\hline $\mathrm{J} 153411.10+543345.2$ & sdOB & $34800 \pm 700$ & $5.64 \pm 0.09$ & $-2.6 \pm 0.3$ & $3.8_{-0.4}^{-0.2}$ & $83 \pm 29$ & 0.018 \\
\hline J082053.53+000843.4 & $\mathrm{sdB}$ & $26700 \pm 900$ & $5.48 \pm 0.10$ & $-2.0 \pm 0.09$ & $1.6_{-0.2}^{+0.4}$ & $77 \pm 11$ & 0.047 \\
\hline $\mathrm{J} 170810.97+244341.6$ & sdOB & $35600 \pm 800$ & $5.58 \pm 0.14$ & $-0.8 \pm 0.1$ & $8.5^{-1.6}$ & $76 \pm 33$ & 0.013 \\
\hline J094856.95+334151.0 & He-sdO & $51000 \pm 1200$ & $5.87 \pm 0.12$ & $+1.8 \pm 0.5$ & $5.1_{-0.7}^{+0.4}$ & $75 \pm 17$ & 0.012 \\
\hline $\mathrm{J} 204613.40-045418.7 \dagger$ & $\mathrm{sdB}$ & $31600 \pm 600$ & $5.55 \pm 0.10$ & $-3.7 \pm 0.6$ & $2.8_{-0.4}^{+0.1}$ & $70 \pm 13$ & 0.030 \\
\hline $\mathrm{J} 215648.71+003620.7$ & $\mathrm{sdB}$ & $30800 \pm 800$ & $5.77 \pm 0.12$ & $-2.2 \pm 0.3$ & $4.7_{-0.7}^{-0.4}$ & $69 \pm 21$ & 0.011 \\
\hline $\mathrm{J} 074534.16+372718.5$ & $\mathrm{sdB}$ & $37500 \pm 500$ & $5.90 \pm 0.09$ & $<-3.0$ & $4.6_{-0.5}^{+0.1}$ & $65 \pm 19$ & 0.036 \\
\hline $\mathrm{J} 143153.05-002824.3$ & sdOB & $37300 \pm 800$ & $6.02 \pm 0.16$ & $-0.8 \pm 0.1$ & $4.4_{-0.8}^{+0.9}$ & $65 \pm 22$ & 0.012 \\
\hline $\mathrm{J} 171629.92+575121.2$ & $\mathrm{sdOB}$ & $35400 \pm 1000$ & $5.60 \pm 0.18$ & $-0.7 \pm 0.1$ & $7.8_{-0.0}^{-1.8}$ & $65 \pm 16$ & 0.013 \\
\hline $\mathrm{J} 112414.45+402637.1$ & $\mathrm{He}-\mathrm{sdO}$ & $47100 \pm 1000$ & $5.81 \pm 0.23$ & $+1.7 \pm 0.7$ & $5.9^{-1.9}$ & $63 \pm 22$ & 0.021 \\
\hline $\mathrm{J} 125702.30+435245.8$ & $\mathrm{sdB}$ & $28000 \pm 1100$ & $5.77 \pm 0.17$ & $<-3.0$ & $4.9_{-1.0}^{+1.4}$ & $63 \pm 28$ & 0.010 \\
\hline $\mathrm{J} 110215.97+521858.1$ & He-sdO & $56600 \pm 4200$ & $5.36 \pm 0.22$ & $>+2.0$ & $8.9_{-22}^{-1.0}$ & $62 \pm 11$ & 0.033 \\
\hline $\mathrm{J} 151415.66-012925.2$ & $\mathrm{He}-\mathrm{sdO}$ & $48200 \pm 500$ & $5.85 \pm 0.08$ & $+1.7 \pm 0.4$ & $3.6_{-03}^{+0.2}$ & $62 \pm 22$ & 0.016 \\
\hline $\mathrm{J} 204300.90+002145.0$ & $\mathrm{sdO}$ & $40200 \pm 700$ & $6.15 \pm 0.13$ & $-1.3 \pm 0.4$ & $3.6_{-0.5}^{+0.3}$ & $61 \pm 13$ & 0.016 \\
\hline $\mathrm{J} 171617.33+553446.7$ & $\mathrm{sdB}$ & $32900 \pm 900$ & $5.48 \pm 0.09$ & $<-3.0$ & $4.9_{-0.6}^{+0.3}$ & $60 \pm 24$ & 0.048 \\
\hline $\mathrm{J} 210454.89+110645.5$ & $\mathrm{sdOB}$ & $37800 \pm 700$ & $5.63 \pm 0.10$ & $-2.4 \pm 0.2$ & $4.9_{-0 .}^{-0.6}$ & $58 \pm 19$ & 0.023 \\
\hline $\mathrm{J} 115358.81+353929.0$ & $\mathrm{sdOB}$ & $29400 \pm 500$ & $5.49 \pm 0.06$ & $-2.5 \pm 0.3$ & $3.3^{-0.0}$ & $56 \pm 12$ & 0.022 \\
\hline $\mathrm{J} 174516.32+244348.3$ & $\mathrm{He}-\mathrm{sdO}$ & $43400 \pm 1000$ & $5.62 \pm 0.21$ & $>+2.0$ & $6.2_{-14}^{-1.8}$ & $55 \pm 28$ & 0.016 \\
\hline $\mathrm{J} 134352.14+394008.3$ & $\mathrm{He}-\mathrm{sdB}$ & $36000 \pm 2100$ & $4.78 \pm 0.30$ & $-0.2 \pm 0.2$ & $\begin{array}{l}8.8_{-61}^{+1.4} \\
8.5\end{array}$ & $52 \pm 34$ & 0.022 \\
\hline $\mathrm{J} 115716.37+612410.7$ & $\mathrm{sdB}$ & $29900 \pm 500$ & $5.59 \pm 0.08$ & $-3.2 \pm 0.8$ & $4.0_{-0.5}^{+0.1}$ & $51 \pm 34$ & 0.049 \\
\hline $\mathrm{J} 133638.81+111949.4$ & $\mathrm{sdB}$ & $27500 \pm 500$ & $5.49 \pm 0.08$ & $-2.7 \pm 0.2$ & $4.4_{-0.5}^{+0.4}$ & $48 \pm 17$ & 0.030 \\
\hline $\mathrm{J} 211651.96+003328.5$ & $\mathrm{sdB}$ & $27900 \pm 800$ & $5.78 \pm 0.15$ & $-3.9 \pm 0.7$ & $4.3_{-0.8}^{+0.9}$ & $48 \pm 23$ & 0.016 \\
\hline $\mathrm{J} 170645.57+243208.6$ & $\mathrm{sdB}$ & $32000 \pm 500$ & $5.59 \pm 0.07$ & $<-4.0$ & $5.5_{-0.5}^{+0.8}$ & $46 \pm 14$ & 0.013 \\
\hline $\mathrm{J} 175125.67+255003.5$ & $\mathrm{sdB}$ & $30600 \pm 500$ & $5.48 \pm 0.08$ & $<-3.8$ & $5.0_{-0.5}^{+0.3}$ & $46 \pm 14$ & 0.034 \\
\hline J012739.35+404357.8 & $\mathrm{sdO}$ & $48300 \pm 3200$ & $5.67 \pm 0.10$ & $-1.3 \pm 0.2$ & $4.1_{-0.6}^{+0.5}$ & $45 \pm 17$ & 0.037 \\
\hline $\mathrm{J} 113418.00+015322.1$ & $\mathrm{sdB}$ & $29700 \pm 1200$ & $4.83 \pm 0.16$ & $<-4.0$ & $1.8_{-2 .}^{+0.6}$ & $45 \pm 24$ & 0.076 \\
\hline $\mathrm{J} 172624.10+274419.3 \dagger$ & $\mathrm{sdOB}$ & $33500 \pm 500$ & $5.71 \pm 0.09$ & $-2.2 \pm 0.1$ & $2.2_{-0.2}^{+0.4}$ & $45 \pm 16$ & 0.047 \\
\hline $\mathrm{J} 155628.34+011335.0$ & $\mathrm{sdB}$ & $32700 \pm 600$ & $5.51 \pm 0.08$ & $-2.9 \pm 0.2$ & $3.1_{-0.4}^{-0.4}$ & $44 \pm 15$ & 0.068 \\
\hline $\mathrm{J} 103549.68+092551.9$ & $\mathrm{He}-\mathrm{sdO}$ & $48100 \pm 600$ & $6.02 \pm 0.13$ & $>+2.0$ & $2.2_{-0.3}^{+0.3}$ & $43 \pm 12$ & 0.021 \\
\hline $\mathrm{J} 141549.05+111213.9$ & $\mathrm{He}-\mathrm{sdO}$ & $43100 \pm 800$ & $5.81 \pm 0.17$ & $>+2.0$ & $2.4_{-0.4}^{+0.5}$ & $43 \pm 7$ & 0.023 \\
\hline $\mathrm{J} 152705.03+110843.9$ & sdOB & $37600 \pm 500$ & $5.62 \pm 0.10$ & $-0.5 \pm 0.1$ & $4.8_{-0.0}^{+0.4}$ & $43 \pm 14$ & 0.054 \\
\hline $\mathrm{J} 052544.93+630726.0$ & sdOB & $35600 \pm 800$ & $5.85 \pm 0.10$ & $-1.6 \pm 0.2$ & $4.3_{-0.5}^{+0.3}$ & $42 \pm 17$ & 0.026 \\
\hline $\mathrm{J} 100535.76+223952.1$ & $\mathrm{sdB}$ & $29000 \pm 700$ & $5.43 \pm 0.13$ & $-2.7 \pm 0.2$ & $7.9_{-13}^{-1.5}$ & $41 \pm 18$ & 0.019 \\
\hline J204546.81-054355.6 & $\mathrm{sdB}$ & $35500 \pm 500$ & $5.47 \pm 0.09$ & $-1.4 \pm 0.2$ & $7.3_{-0.8}^{+0.3}$ & $41 \pm 18$ & 0.013 \\
\hline J092520.70+470330.6 & $\mathrm{sdB}$ & $28100 \pm 900$ & $5.17 \pm 0.15$ & $-2.5 \pm 0.2$ & $7.5_{-14}^{-0.8}$ & $40 \pm 13$ & 0.012 \\
\hline $\mathrm{J} 075937.15+541022.2$ & $\mathrm{sdB}$ & $31300 \pm 700$ & $5.30 \pm 0.10$ & $-3.3 \pm 0.3$ & $7.6^{-1.1}+1$ & $38 \pm 13$ & 0.012 \\
\hline $\mathrm{J} 234528.85+393505.2$ & He-sdO & $47900 \pm 800$ & $6.07 \pm 0.14$ & $>+2.0$ & $3.5_{-0.5}^{+0.0}$ & $37 \pm 14$ & 0.012 \\
\hline $\mathrm{J} 130439.57+312904.8$ & sdOB & $38100 \pm 600$ & $5.69 \pm 0.12$ & $-0.4 \pm 0.1$ & $4.1_{-0.6}^{-0.5}$ & $36 \pm 12$ & 0.037 \\
\hline $\mathrm{J} 130059.20+005711.7 \ddagger$ & He-sdO & $40700 \pm 500$ & $5.53 \pm 0.10$ & $-0.6 \pm 0.1$ & $3.9_{-0.4}^{-0.6}$ & $36 \pm 16$ & 0.012 \\
\hline $\mathrm{J} 110445.01+092530.9$ & sdOB & $35900 \pm 800$ & $5.41 \pm 0.07$ & $-2.1 \pm 0.4$ & $3.8_{-0.3}^{-0.4}$ & $34 \pm 14$ & 0.040 \\
\hline $\mathrm{J} 113935.45+614953.9$ & $\mathrm{sdB}$ & $28800 \pm 900$ & $5.27 \pm 0.15$ & $-2.8 \pm 0.3$ & $4.9_{-09}^{+1.1}$ & $31 \pm 14$ & 0.011 \\
\hline $\mathrm{J} 233406.11+462249.3$ & sdOB & $34600 \pm 500$ & $5.71 \pm 0.09$ & $-1.3 \pm 0.1$ & $4.9_{-0 .}^{-0.9}$ & $31 \pm 14$ & 0.025 \\
\hline $\mathrm{J} 225638.34+065651.1 \dagger$ & $\mathrm{sdB}$ & $28900 \pm 600$ & $5.58 \pm 0.11$ & $-3.0 \pm 0.2$ & $1.6_{-02}^{-0.0}$ & $27 \pm 11$ & 0.031 \\
\hline $\mathrm{J} 140545.25+014419.0$ & $\mathrm{sdB}$ & $27300 \pm 800$ & $5.37 \pm 0.16$ & $-1.9 \pm 0.2$ & $2.5_{-0.5}^{+0.2}$ & $25 \pm 10$ & 0.026 \\
\hline
\end{tabular}

Notes. $\uparrow$ The binary system has been analysed in Geier et al. (2011). $\$$ Atmospheric parameters $\left(T_{\text {eff }}=39400 \mathrm{~K}, \log g=5.64, \log y=-0.55\right)$ have been determined by Ströer et al. (2007). 
S. Geier et al.: The MUCHFUSS project - searching for hot subdwarf binaries with massive unseen companions

Appendix A: Close binary subdwarfs from literature

Table A.1. Orbital parameters of all known hot subdwarf binaries from literature.

\begin{tabular}{|c|c|c|c|c|}
\hline 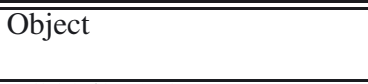 & $\begin{array}{c}P \\
\text { [d] }\end{array}$ & $\begin{array}{c}\gamma \\
{\left[\mathrm{km} \mathrm{s}^{-1}\right]}\end{array}$ & $\begin{array}{c} \\
{\left[\mathrm{km} \mathrm{s}^{-1}\right]} \\
\end{array}$ & $\overline{\text { Reference }}$ \\
\hline PG 0850+170 & 27.815 & $32.2 \pm 2.8$ & $33.5 \pm 3.3$ & Morales-Rueda et al. (2003a) \\
\hline PG $1619+522$ & 15.3578 & $-52.5 \pm 1.1$ & $35.2 \pm 1.1$ & Morales-Rueda et al. (2003a) \\
\hline PG 1110+294 & 9.4152 & $-15.2 \pm 0.9$ & $58.7 \pm 1.2$ & Morales-Rueda et al. (2003a) \\
\hline Feige 108 & 8.7465 & $45.8 \pm 0.6$ & $50.2 \pm 1.0$ & Edelmann et al. (2004) \\
\hline PG 0940+068 & 8.330 & $-16.7 \pm 1.4$ & $61.2 \pm 1.4$ & Maxted et al. (2000b) \\
\hline PHL 861 & 7.44 & $-26.5 \pm 0.4$ & $47.9 \pm 0.4$ & Karl et al. (2006) \\
\hline HE $1448-0510$ & 7.159 & $-45.5 \pm 0.8$ & $53.7 \pm 1.1$ & Karl et al. (2006) \\
\hline PG $1032+406$ & 6.7791 & $24.5 \pm 0.5$ & $33.7 \pm 0.5$ & Morales-Rueda et al. (2003a) \\
\hline PG $0907+123$ & 6.11636 & $56.3 \pm 1.1$ & $59.8 \pm 0.9$ & Morales-Rueda et al. (2003a) \\
\hline HE $1115-0631$ & 5.87 & $87.1 \pm 1.3$ & $61.9 \pm 1.1$ & Napiwotzki et al. (in prep.) \\
\hline $\mathrm{CD}-24731$ & 5.85 & $20.0 \pm 5.0$ & $63.0 \pm 3.0$ & Edelmann et al. (2005) \\
\hline PG $1244+113$ & 5.75207 & $9.8 \pm 1.2$ & $55.6 \pm 1.8$ & Morales-Rueda et al. (2003b) \\
\hline PG 0839+399 & 5.6222 & $23.2 \pm 1.1$ & $33.6 \pm 1.5$ & Morales-Rueda et al. (2003a) \\
\hline TON S 135 & 4.1228 & $-3.7 \pm 1.1$ & $41.4 \pm 1.5$ & Edelmann et al. (2005) \\
\hline PG 0934+186 & 4.051 & $7.4 \pm 2.9$ & $60.2 \pm 2.0$ & Morales-Rueda et al. (2003b) \\
\hline PB 7352 & 3.62166 & $-2.1 \pm 0.3$ & $60.8 \pm 0.3$ & Edelmann et al. (2005) \\
\hline KPD $0025+5402$ & 3.5711 & $-7.8 \pm 0.7$ & $40.2 \pm 1.1$ & Morales-Rueda et al. (2003a) \\
\hline TON 245 & 2.501 & - & 88.3 & Morales-Rueda et al. (2003a) \\
\hline PG $1300+2756$ & 2.25931 & $-3.1 \pm 0.9$ & $62.8 \pm 1.6$ & Morales-Rueda et al. (2003a) \\
\hline NGC 188/II-91 & 2.15 & - & 22.0 & Green et al. (2004) \\
\hline V 1093 Her $^{\mathrm{p}}$ & 1.77732 & $-3.9 \pm 0.8$ & $70.8 \pm 1.0$ & Morales-Rueda et al. (2003a) \\
\hline HD 171858 & 1.63280 & $62.5 \pm 0.1$ & $60.8 \pm 0.3$ & Edelmann et al. (2005) \\
\hline KPD 2040+3954 & 1.48291 & $-11.5 \pm 1.0$ & $95.1 \pm 1.7$ & Morales-Rueda et al. (2003b) \\
\hline HE 2150-0238 & 1.321 & $-32.5 \pm 0.9$ & $96.3 \pm 1.4$ & Karl et al. (2006) \\
\hline [CW83] 1735+22 & 1.278 & $20.6 \pm 0.4$ & $103.0 \pm 1.5$ & Edelmann et al. (2005) \\
\hline PG $1512+244$ & 1.26978 & $-2.9 \pm 1.0$ & $92.7 \pm 1.5$ & Morales-Rueda et al. (2003a) \\
\hline PG 0133+114 & 1.23787 & $-0.3 \pm 0.2$ & $82.0 \pm 0.3$ & Edelmann et al. (2005) \\
\hline HE 1047-0436 & 1.21325 & $25.0 \pm 3.0$ & $94.0 \pm 3.0$ & Napiwotzki et al. (2001) \\
\hline HE $1421-1206$ & 1.188 & $-86.2 \pm 1.1$ & $55.5 \pm 2.0$ & Napiwotzki et al. (in prep.) \\
\hline PG $1000+408$ & 1.041145 & 41.9 & 72.4 & Shimanskii et al. (2008) \\
\hline PB 5333 & 0.92560 & $-95.3 \pm 1.3$ & $22.4 \pm 0.8$ & Edelmann et al. (2004) \\
\hline HE 2135-3749 & 0.9240 & $45.0 \pm 0.5$ & $90.5 \pm 0.6$ & Karl et al. (2006) \\
\hline EC $12408-1427$ & 0.90243 & $-52.0 \pm 1.2$ & $58.9 \pm 1.6$ & Morales-Rueda et al. (2006) \\
\hline PG 0918+0258 & 0.87679 & $104.4 \pm 1.7$ & $80.0 \pm 2.6$ & Morales-Rueda et al. (2003a) \\
\hline PG $1116+301$ & 0.85621 & $-0.2 \pm 1.1$ & $88.5 \pm 2.1$ & Morales-Rueda et al. (2003a) \\
\hline PG $1230+052$ & 0.8372 & $-43.4 \pm 0.8$ & $41.5 \pm 1.3$ & Morales-Rueda et al. (2003b) \\
\hline $\mathrm{V} 2579 \mathrm{Oph}^{\mathrm{p}}$ & 0.8292056 & $-54.16 \pm 0.27$ & $70.10 \pm 0.13$ & For et al. (2006) \\
\hline TON S 183 & 0.8277 & $50.5 \pm 0.8$ & $84.8 \pm 1.0$ & Edelmann et al. (2005) \\
\hline EC 02200-2338 & 0.8022 & $20.7 \pm 2.3$ & $96.3 \pm 1.4$ & Morales-Rueda et al. (2005) \\
\hline PG 0849+319 & 0.74507 & $64.0 \pm 1.5$ & $66.3 \pm 2.1$ & Morales-Rueda et al. (2003a) \\
\hline $\mathrm{JL} 82^{\mathrm{r}}$ & 0.73710 & $-1.6 \pm 0.8$ & $34.6 \pm 1.0$ & Edelmann et al. (2005) \\
\hline PG $1248+164$ & 0.73232 & $-16.2 \pm 1.3$ & $61.8 \pm 1.1$ & Morales-Rueda et al. (2003a) \\
\hline HD $188112 \dagger$ & 0.60658125 & $26.6 \pm 0.3$ & $188.4 \pm 0.2$ & Edelmann et al. (2005) \\
\hline PG $1247+554$ & 0.602740 & $13.8 \pm 0.6$ & $32.2 \pm 1.0$ & Maxted et al. (2000b) \\
\hline PG $1725+252$ & 0.601507 & $-60.0 \pm 0.6$ & $104.5 \pm 0.7$ & Morales-Rueda et al. (2003a) \\
\hline PG $0101+039^{\text {el,p }}$ & 0.569899 & $7.3 \pm 0.2$ & $104.7 \pm 0.4$ & Geier et al. (2008) \\
\hline HE 1059-2735 & 0.555624 & $-44.7 \pm 0.6$ & $87.7 \pm 0.8$ & Napiwotzki et al. (in prep.) \\
\hline PG $1519+640$ & 0.54029143 & $0.1 \pm 0.4$ & $42.7 \pm 0.6$ & Edelmann et al. (2004) \\
\hline PG $0001+275$ & 0.529842 & $-44.7 \pm 0.5$ & $92.8 \pm 0.7$ & Edelmann et al. (2005) \\
\hline PG $1743+477$ & 0.515561 & $-65.8 \pm 0.8$ & $121.4 \pm 1.0$ & Morales-Rueda et al. (2003a) \\
\hline HE $1318-2111$ & 0.487502 & $48.9 \pm 0.7$ & $48.5 \pm 1.2$ & Napiwotzki et al. (in prep.) \\
\hline PG $1544+488 \ddagger$ & 0.48 & $-23 \pm 4$ & $57 \pm 4 / 97 \pm 10$ & Ahmad et al. (2004) \\
\hline GALEX J234947.7+384440 & 0.46249 & $2.0 \pm 1.0$ & $87.9 \pm 2.2$ & Kawka et al. (2010) \\
\hline HE $0230-4323^{r, p}$ & 0.45152 & $16.6 \pm 1.0$ & $62.4 \pm 1.6$ & Edelmann et al. (2005) \\
\hline HE 0929-0424 & 0.4400 & $41.4 \pm 1.0$ & $114.3 \pm 1.4$ & Karl et al. (2006) \\
\hline
\end{tabular}


Table A.1. continued.

\begin{tabular}{|c|c|c|c|c|}
\hline$\overline{\text { Object }}$ & $\begin{array}{c}P \\
\text { [d] } \\
\end{array}$ & $\begin{array}{c}\gamma \\
{\left[\mathrm{km} \mathrm{s}^{-1}\right]}\end{array}$ & $\begin{array}{c}K \\
{\left[\mathrm{~km} \mathrm{~s}^{-1}\right]}\end{array}$ & $\overline{\overline{\text { Reference }}}$ \\
\hline [CW83] 1419-09 & 0.4178 & $42.3 \pm 0.3$ & $109.6 \pm 0.4$ & Edelmann et al. (2005) \\
\hline KPD $1946+4340^{\mathrm{ec}, \mathrm{el}}$ & 0.403739 & $-5.5 \pm 1.0$ & $167.0 \pm 2.4$ & Morales-Rueda et al. (2003a) \\
\hline KUV $04421+1416^{\mathrm{r}, \mathrm{p}}$ & 0.398 & $33 \pm 3$ & $90 \pm 5$ & Reed et al. (2010) \\
\hline Feige $48^{p}$ & 0.376 & $-47.9 \pm 0.1$ & $28.0 \pm 0.2$ & O'Toole et al. (2004) \\
\hline GD 687 & 0.37765 & $32.3 \pm 3.0$ & $118.3 \pm 3.4$ & Geier et al. (2010a) \\
\hline PG 1232-136 & 0.3630 & $4.1 \pm 0.3$ & $129.6 \pm 0.04$ & Edelmann et al. (2005) \\
\hline PG $1101+249$ & 0.35386 & $-0.8 \pm 0.9$ & $134.6 \pm 1.3$ & Moran et al. (1999) \\
\hline PG $1438-029^{r}$ & 0.336 & - & 32.1 & Green et al. (2005) \\
\hline PG $1528+104$ & 0.331 & $-49.9 \pm 0.8$ & $52.7 \pm 1.3$ & Morales-Rueda et al. (2003b) \\
\hline PG $0941+280^{\text {ec }}$ & 0.315 & - & - & Green et al. (2004) \\
\hline $\operatorname{KBS} 13^{\mathrm{r}}$ & 0.2923 & $7.53 \pm 0.08$ & $22.82 \pm 0.23$ & For et al. (2008) \\
\hline CPD-64 481 & 0.2772 & $94.1 \pm 0.3$ & $23.8 \pm 0.4$ & Edelmann et al. (2005) \\
\hline GALEX J032139.8+472716 & 0.26584 & $70.5 \pm 2.2$ & $59.8 \pm 4.5$ & Kawka et al. (2010) \\
\hline HE $0532-4503$ & 0.2656 & $8.5 \pm 0.1$ & $101.5 \pm 0.2$ & Karl et al. (2006) \\
\hline AA Dor ${ }^{\mathrm{ec}, \mathrm{r}}$ & 0.2614 & $1.57 \pm 0.09$ & $40.15 \pm 0.11$ & Müller et al. (2010) \\
\hline PG $1329+159^{r}$ & 0.249699 & $-22.0 \pm 1.2$ & $40.2 \pm 1.1$ & Morales-Rueda et al. (2003a) \\
\hline PG $2345+318^{\text {ec }}$ & 0.2409458 & $-10.6 \pm 1.4$ & $141.2 \pm 1.1$ & Moran et al. (1999) \\
\hline PG $1432+159$ & 0.22489 & $-16.0 \pm 1.1$ & $120.0 \pm 1.4$ & Moran et al. (1999) \\
\hline BPS CS 22169-0001 ${ }^{\mathrm{r}}$ & 0.1780 & $2.8 \pm 0.3$ & $14.9 \pm 0.4$ & Edelmann et al. (2005) \\
\hline HS $2333+3927^{r}$ & 0.1718023 & $-31.4 \pm 2.1$ & $89.6 \pm 3.2$ & Heber et al. (2004) \\
\hline $2 \mathrm{M} 1533+3759^{\mathrm{ec}, \mathrm{r}}$ & 0.16177042 & $-3.4 \pm 5.2$ & $71.1 \pm 1.0$ & For et al. (2010) \\
\hline EC 00404-4429 & 0.12834 & $33.0 \pm 2.9$ & $152.8 \pm 3.4$ & Morales-Rueda et al. (2005) \\
\hline $2 \mathrm{M} 1938+4603^{\mathrm{ec}, \mathrm{r}}$ & 0.1257653 & $20.1 \pm 0.3$ & $65.7 \pm 0.6$ & Østensen et al. (2010) \\
\hline BUL-SC $16335^{\mathrm{ec}, \mathrm{r}}$ & 0.125050278 & - & - & Polubek et al. (2007) \\
\hline PG $1043+760$ & 0.1201506 & $24.8 \pm 1.4$ & $63.6 \pm 1.4$ & Morales-Rueda et al. (2003a) \\
\hline HW Virec,r & 0.115 & $-13.0 \pm 0.8$ & $84.6 \pm 1.1$ & Edelmann (2008) \\
\hline HS $2231+2441^{\mathrm{ec}, \mathrm{r}}$ & 0.1105880 & - & $49.1 \pm 3.2$ & Østensen et al. (2007) \\
\hline NSVS $14256825^{\mathrm{ec}, \mathrm{r}}$ & 0.110374102 & - & - & Wils et al. (2007) \\
\hline PG $1336-018^{\mathrm{ec}, \mathrm{r}, \mathrm{p}}$ & 0.101015999 & -25.0 & $78.7 \pm 0.6$ & Vučković et al. (2007) \\
\hline HS $0705+6700^{\mathrm{ec}, \mathrm{r}}$ & 0.09564665 & $-36.4 \pm 2.9$ & $85.8 \pm 3.6$ & Drechsel et al. (2001) \\
\hline KPD $1930+2752^{\mathrm{el}, \mathrm{p}}$ & 0.0950933 & $5.0 \pm 1.0$ & $341.0 \pm 1.0$ & Geier et al. (2007) \\
\hline $\mathrm{KPD} 0422+5421^{\mathrm{ec}, \mathrm{el}}$ & 0.09017945 & $-57.0 \pm 12.0$ & $237.0 \pm 18.0$ & Orosz \& Wade (1999) \\
\hline $\mathrm{NGC} 6121-\mathrm{V} 46^{\mathrm{el}} \dagger$ & 0.087159 & $31.3 \pm 1.6$ & $211.6 \pm 2.3$ & O'Toole et al. (2006) \\
\hline PG $1017-086^{r}$ & 0.0729938 & $-9.1 \pm 1.3$ & $51.0 \pm 1.7$ & Maxted et al. (2002) \\
\hline
\end{tabular}

Notes. The superscript $\mathrm{p}$ denotes sdB pulsators, $r$ binaries where with reflection effect, ec eclipsing systems and el systems with light variations caused by ellipsoidal deformation. $\dagger$ Post-RGB stars without core helium-burning. $\ddagger$ Double-lined binary consisting of two helium rich sdBs. The RV semi-amplitudes of both components are given. 